\title{
Nerve-Specific Input Modulation to Spinal Neurons during a Motor Task in the Monkey
}

\author{
Joachim Confais, ${ }^{1 *}$ Geehee Kim, ${ }^{1,2 *}$ Saeka Tomatsu, ${ }^{1}{ }^{\oplus T o m o h i k o ~ T a k e i, ~}{ }^{1,2}$ and ${ }^{\circ}$ Kazuhiko Seki ${ }^{1,2,3}$ \\ ${ }^{1}$ Department of Neurophysiology, National Institute of Neuroscience, Tokyo 187-8502, Japan, ${ }^{2}$ Department of Developmental Physiology, National Institute \\ for Physiological Sciences, Okazaki 444-8585, Japan, and ${ }^{3}$ Precursory Research for Embryonic Science and Technology, Japan Science and Technology \\ Agency, Saitama 332-0012, Japan
}

If not properly regulated, the large amount of reafferent sensory signals generated by our own movement could destabilize the CNS. We investigated how input from peripheral nerves to spinal cord is modulated during behavior. We chronically stimulated the deep radial nerve (DR; proprioceptive, wrist extensors), the median nerve (M; mixed, wrist flexors and palmar skin) and the superficial radial nerve (SR; cutaneous, hand dorsum) while four monkeys performed a delayed wrist flexion- extension task. Spinal neurons putatively receiving direct sensory input were defined based on their evoked response latency following nerve stimulation. We compared the influence of behavior on the evoked response (responsiveness to a specific peripheral input) and firing rate of 128 neuron-nerve pairs based on their source nerve. Firing rate increased during movement regardless of source nerve, whereas evoked response modulation was strikingly nerve-dependent. In SR $(n=47)$ and M $(n=27)$ neurons (cutaneous or mixed input), the evoked response was suppressed during wrist flexion and extension. In contrast, in DR neurons ( $n=54$, pure proprioceptive input), the evoked response was facilitated exclusively during movements corresponding to the contraction of DR spindle-bearing muscles (i.e., wrist extension). Furthermore, modulations of firing rate and evoked response were uncorrelated in SR and M neurons, whereas they tended to be positively comodulated in DR neurons. Our results suggest that proprioceptive and cutaneous inputs to the spinal cord are modulated differently during voluntary movements, suggesting a refined gating mechanism of sensory signals according to behavior.

Key words: nerve stimulation; primates; sensory gating; somatosensory; spinal neurons; voluntary movement

\section{Significance Statement}

Voluntary movements produce copious sensory signals, which may overwhelm the CNS if not properly regulated. This regulation is called "gating" and occurs at several levels of the CNS. To evaluate the specificity of sensory gating, we investigated how different sources of somatosensory inputs to the spinal cord were modulated while monkeys performed wrist movements. We recorded activity from spinal neurons that putatively received direct connections from peripheral nerves while stimulating their source nerves, and measured the evoked responses. Whereas cutaneous inputs were suppressed regardless of the type of movement, muscular inputs were specifically facilitated during relevant movements. We conclude that, even at the spinal level, sensory gating is a refined and input-specific process.

\section{Introduction}

Motor behavior stimulates proprioceptive and cutaneous receptors, generating a large inflow of sensory information. These

Received Aug. 11, 2016; revised Jan. 25, 2017; accepted Jan. 30, 2017

Author contributions: K.S. designed research; G.K., T.T., and K.S. performed research; J.C., G.K., and S.T. analyzed data; J.C. and K.S. wrote the paper.

This work was supported in part by National Institutes of Health Grants NS12542, NS36781, and RR00166; Human Frontiers Science Program Grant LT0070/1999-B; Grants-in-Aid 18020030, 18047027, and 26120003 for Scientific Research on Priority Areas, Mobilligence, System Study on Higher-order Brain Function, Innovative Areas Understanding Brain Plasticity on Body Representations to Promote their Adaptive Functions from the Ministry of Education, Culture, Sports, Science and Technology of Japan to K.S.; and the Japan Science and Technology Agency Precursory Research for Embryonic Science and Technology programs to K.S. We thank Nobuaki Takahashi and Morio Togawa (National Institute for Physiological Sciences) for technical assistance; Eberhard E. Fetz and Steve I. Perl- time-varying reafferent signals are transmitted via sensory nerves to spinal or subcortical networks, and then to the rest of the CNS. The way in which this large amount of self-generated sensory information interacts with descending motor commands with-

mutter (University of Washington, Seattle) for permission to use the data on spinal INs with SR input; and Roland Philipp for comments on the manuscript.

The authors declare no competing financial interests.

*J.C. and G.K. contributed equally to this study.

Correspondence should be addressed to Dr. Kazuhiko Seki, Department of Neurophysiology, National Institute of Neuroscience, 4-1-1 Ogawa-Higashi, Kodaira, Tokyo 187-8502, Japan. E-mail: seki@ncnp.go.jp.

T. Takei's present address: Centre for Neuroscience Studies, Queen's University, Kingston, Ontario K7L 3N6, Canada.

DOI:10.1523/JNEUROSCI.2561-16.2017

Copyright $\odot 2017$ the authors $\quad 0270-6474 / 17 / 372612-15 \$ 15.00 / 0$ 
out having an overwhelming effect has been a long-standing question in neuroscience (Rudomín, 2002).

This question is related to the concept of "sensory gating": the regulation of sensory inputs at different levels of the CNS to keep them at a manageable scale (Rushton et al., 1981). Indeed, during movement, sensory inputs are generally depressed compared with those at rest, and this suppression is modulated by movement parameters (e.g., velocity) (Ghez and Pisa, 1972). In humans, cutaneous perception decreases during finger movements (Angel and Malenka, 1982; Bays et al., 2006), and both cutaneous reflexes and the H-reflex decrease with the step cycle (Capaday and Stein, 1987b; Baken et al., 2006).

Correspondingly, spinal and cortical responses to cutaneous stimulation decrease in amplitude (Chapin et al., 1982; Jiang et al., 1990, 1991) during movement. Sensory gating can, however, occur at several levels along the CNS (Ghez and Pisa, 1972; Chapman et al., 1988), reflecting potentially parallel mechanisms (Seki and Fetz, 2012; Palmer et al., 2016). It is therefore difficult to estimate the locus and mechanisms of sensory gating based solely on investigations of behavioral output (e.g., reflexes) or neuronal activity at relatively late stages of sensory processing (e.g., cortex).

Seki et al. (2003) focused on the "entry door" of the sensory system: the so-called first-order spinal interneurons (FOINs), which receive monosynaptic connections from peripheral nerves. As these neurons are the first relay sites of peripheral receptors to spinal and supraspinal centers, their activity is expected to influence all later stages of sensorimotor control. The researchers observed a dynamic decrease in FOIN responses to cutaneous nerve stimulation during movement. At least part of this modulation seemed to occur upstream from the FOINs; that is, the cutaneous volley was presynaptically suppressed before it reached the FOINs (Seki et al., 2009). This result shed some light on the early gating of cutaneous afferents during movement. However, this phenomenon could be the result of (1) a general suppression of all peripheral inputs during movement, (2) a more targeted suppression of cutaneous information coming from a specific input (e.g., a specific patch of skin), or (3) any intermediate degree between these two extremes. For example, both cutaneous (Angel and Malenka, 1982) and muscular sensations (Collins et al., 1998) decrease during movement, which could be interpreted as a general input suppression. Conversely, distinct populations of spinal interneurons mediate the suppression of inputs from different types of afferent fibers (Rudomín and Schmidt, 1999), implicating a modality-dependent input control. Such differential control would enable fine-tuned regulation of sensory reafferent signals during behavior.

This is the question that the current paper addresses directly: how are spinal inputs from different sensory sources modulated during movement? In other words, does the sensory gating that accompanies voluntary movement depend on the nature (or receptive fields) of afferent signals?

We thus extended the work of Seki et al. (2003) by recording not only from spinal interneurons that received projections from a cutaneous nerve but also from neurons with inputs from two other forearm nerves. We assessed neuronal responses to stimulation of these three nerves, which diverged based on their receptive field (dorsum or palm of the hand) and modality (purely cutaneous, purely proprioceptive, and mixed) while monkeys performed a wrist movement task. We show that input modulation depends on the signal source, task epoch, and movement direction. This result emphasizes the selective and dynamic aspects of a fine-tuned sensory gating control mechanism, by which the CNS shapes incoming information according to behavior.
These results have already been presented in abstract form (Confais et al., 2015).

\section{Materials and Methods}

Animals

These experiments were approved by the Institutional Animal Care and Use Committee of the National Institute for Physiological Sciences and the Institutional Animal Care and Use Committee at the University of Washington. We obtained data from three male Macaca fuscata (Monkeys KO, IS, OK) and one male Macaca nemestrina (Monkey KJ). Data from Monkey KJ were recorded in the laboratory of Dr. Eberhard E. Fetz and used with his consent, and data from the other three animals were recorded in National Institute for Physiological Sciences, Okazaki, Japan. During training and recording sessions, each monkey sat upright in a primate chair with its right arm restrained and its elbow bent at $90^{\circ}$. The monkey's hand was held in a cast, with fingers extended and wrist in the mid-supination/pronation position. The cast holding the monkey's hand was attached to a servomotor-driven manipulandum that measured flexion-extension torque about the wrist. The left arm was loosely restrained to the chair (Seki et al., 2003, 2009; Seki and Fetz, 2012).

\section{Behavioral paradigm}

The monkeys performed a wrist flexion-extension task with an instructed delay period (Prut and Fetz, 1999; Seki et al., 2003, 2012), as illustrated in Figure 1A. A wrist flexion-extension torque applied to the spring-loaded manipulandum controlled the position of a cursor displayed on a computer monitor in front of the monkey. Because the monkeys performed the task with their right hand, a wrist flexion led to a leftward displacement of the cursor. Trials began with the monkey holding the cursor in a center target window corresponding to zero torque for 1.3-1.6 s (rest). Next, flexion and extension targets (empty rectangles) were shown to the left and right of the center target. One target flashed briefly for $0.3 \mathrm{~s}$ (cue), indicating the correct movement to be performed at the end of the instructed delay period, which was signaled by the disappearance of the center target (GO). Trials were accepted only if no wrist movement occurred during the delay period (1.5-2 s). Following the GO signal, the monkey quickly moved (active movement) the cursor to the desired target $(<1.5 \mathrm{~s}$ including reaction time) and held the cursor in the target window for a period of $1.5 \mathrm{~s}$ (active hold). The movements were made against an elastic load applied by the servomotor $(5 \mathrm{~N} \times \mathrm{m})$. At the end of the active hold period, the peripheral target disappeared, and the center target reappeared (release GO). The monkey then relaxed its forearm muscles, allowing the servo-spring to passively return the wrist ( passive movement) to the zero torque position (rest). After keeping the cursor within the center target for $0.8 \mathrm{~s}$, the monkey was rewarded with apple sauce (reward) for successful trials. In each trial, the five epochs marked in blue in Figure $1 A$ were used for analysis (rest, delay, active movement, active hold, and passive movement).

\section{Surgical implants}

Following behavioral training, surgeries were performed aseptically after placing the animals $<1.5 \%-3.0 \%$ sevoflurane anesthesia with a $2: 1$ ratio of $\mathrm{O}_{2}: \mathrm{N} 2 \mathrm{O}$. Head stabilization lugs were cemented to the skull with dental acrylic and anchored to the bone via screws. A stainless-steel recording chamber was implanted over a hemi-laminectomy in the lower cervical vertebrae (C4-C7). Pairs of stainless steel wires (AS632, Cooner Wire) were implanted subcutaneously in 10-12 muscles: extensor carpi ulnaris (ECU), extensor carpi radialis (ECR), extensor digitorum communis (EDC), extensor digitorum-2,3 (ED23), extensor digitorum-4,5 (ED45), flexor carpi radialis (FCR), flexor carpi ulnaris (FCU), flexor digitorum superficialis (FDS), palmaris longus (PL), pronator teres (PT), abductor pollicis longus (APL), and brachioradialis (BRD). These muscles were active in one or both directions. Each muscle was identified based on its anatomical location and characteristic movements, elicited by trains of low-intensity intramuscular stimulation. Nerve cuff electrodes (Haugland, 1996) were implanted on the median (M) and radial nerves for stimulation (Fig. 1B). For the median nerve, the cuff was implanted $\sim 2 \mathrm{~cm}$ proximal to the elbow joint. For the radial nerve, three cuff electrodes were implanted. Two cuffs were implanted on the cutaneous 
A
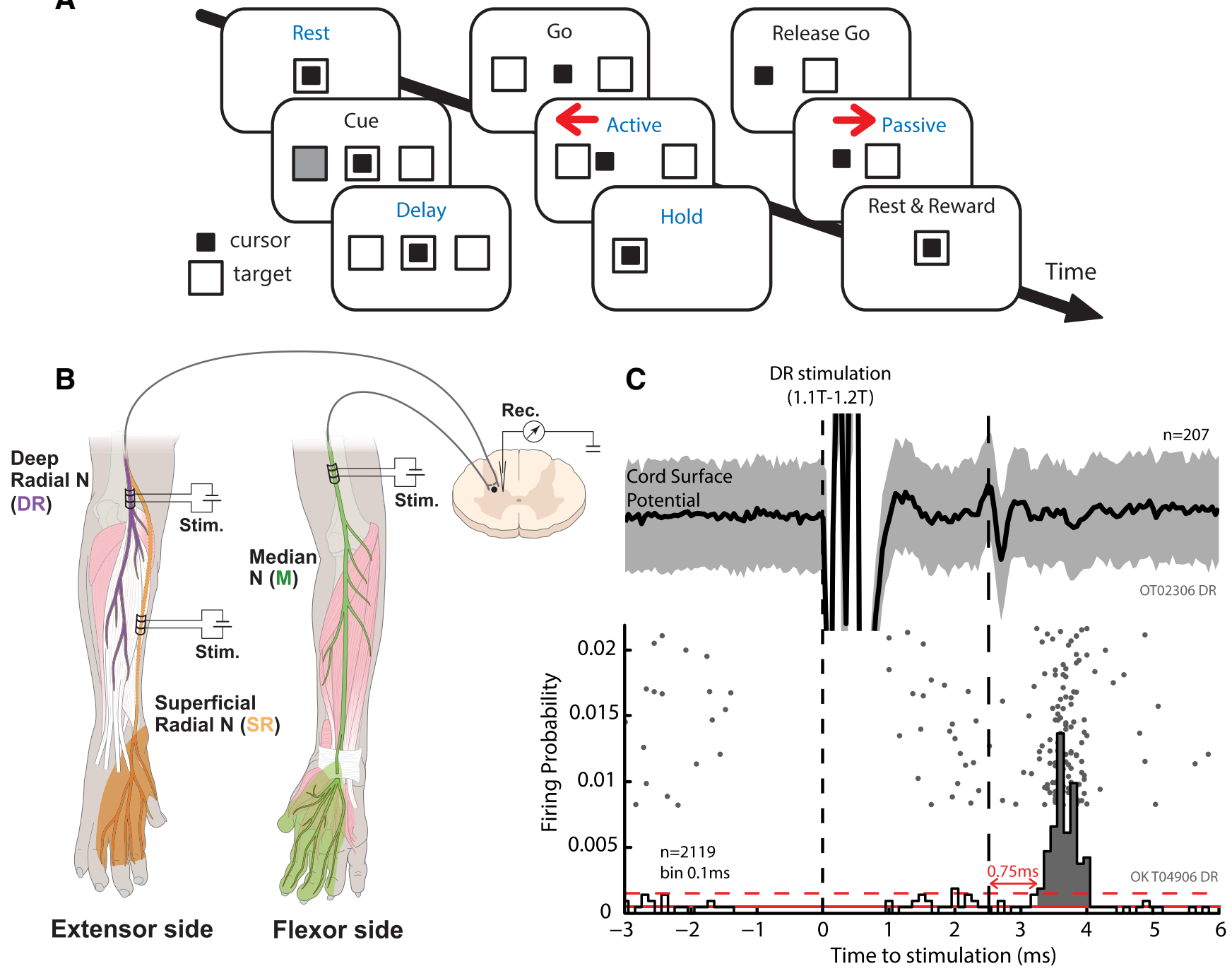

Figure 1. Task sequence and nerve stimulation method. $A$, Task sequence. Black filled square represents a moving cursor. Solid empty squares represent central and peripheral targets. Gray filled square represents the peripheral cue. Red arrows indicate movement direction. Epochs marked in blue represent those analyzed in the present report. Active, Active movement; passive, passive movement. During flexion trials, the active movement and hold epochs required a wrist flexion movement, and the passive movement involved a wrist extension (and vice versa for extension trials). For more information, see Materials and Methods. $\boldsymbol{B}$, Schematics of the experimental setup. Nerve cuff electrodes were chronically implanted on three peripheral nerves of the arm: the deep branch of the radial nerve (DR, purple), the superficial branch of the radial nerve ( $S R$, orange), and the median nerve ( $M$, green). Another cuff electrode was implanted in the $S R$ nerve to record the incoming volley (proximal to that for stimulation; data not shown). DR mostly projects to extensor muscles in the forearm (ECR, ECU, EDC, ED23, ED45, APL, extensor pollicis longus, supinator). SR exclusively innervates a patch of skin on the dorsal, radial aspect of the hand. M projects to flexor muscles of the wrist (PT, FCR, PL, FDS, flexor digitorum profundus [radial side], flexor pollicis longus, abductor pollicis brevis, pronator quadratus) and also innervates a patch of palmar skin on the radial side. Concurrently to the nerve stimulation, the activity of spinal neurons was recorded extracellularly using single metal electrodes. C, Calculation of the evoked peak area. In this figure, the 0 point (short dashed vertical line) indicates the timing of nerve stimulation (in this example, DR). Top, Averaged trace of the CSP, with the SDs of individual traces shown as shaded areas. Here, the first peak of the incoming volley is visible $2.55 \mathrm{~ms}$ after stimulation (long dashed line). Bottom, Raster plot and PSTH of a single spinal neuron. In the raster plot, each row represents the period surrounding a stimulation pulse and each dot represents an action potential. The PSTH summarizes the firing profile of the neuron in response to stimulation, with a bin size of $0.1 \mathrm{~ms}$. Solid horizontal red line indicates the mean firing rate during the $50 \mathrm{~ms}$ preceding stimulation. Dashed red line illustrates 2 SDs above the mean. Filled gray area represents the evoked peak area, which starts and ends when the PSTH crosses the dashed red line. Only the area above baseline (solid red line) is filled in gray. The latency between the incoming volley and the start of the evoked peak reflects the central latency, illustrated by a red arrow. Neurons with a central latency of $<1.5 \mathrm{~ms}$ were classified as "short-latency neurons." For more information, see Materials and Methods.

branch (superficial radial nerve [SR]: approximately midway between the elbow and wrist) and one was implanted on the muscle branch (deep radial nerve $[D R]: \sim 2 \mathrm{~cm}$ proximal to the elbow joint). In the $S R$, the stimulating cuff was implanted distally, and the recording cuff (for incoming volleys) was implanted proximally (Seki et al., 2009). Nerve cuffs were implanted on the M, SR, and DR in Monkeys KO, IS, and OK. For Monkey KJ, cuffs were implanted on the SR only.

\section{Data analysis}

Identification of short-latency neurons (SLNs). Glass-insulated Tungsten or Elgiloy microelectrodes (impedance $0.8-1.4 \mathrm{M} \Omega$ ) were used to record neuronal activity. At the beginning of each electrode penetration, cord surface potentials (CSPs) were monitored, and threshold currents that evoked an incoming volley from the SR, DR, and M were measured on most recording days. The threshold for SR was mainly determined by monitoring the incoming volleys evoked at the proximal cuff. Subsequently, spinal interneuron (IN) single-unit responses were examined by stimulating peripheral nerves with biphasic constant-current pulses (100 $\mu \mathrm{s} /$ phase) at a constant frequency of $1-2 \mathrm{~Hz}$ for Monkeys KO, IS, and $\mathrm{OK}$, and $3 \mathrm{~Hz}$ for Monkey KJ. The stimulus current was set at 1-1.2 times the threshold for DR and M, and 2 times the threshold for SR. Each action potential was isolated based on its waveform, and peristimulus time histograms (PSTHs) were obtained for each IN. The segmental 
response latency was calculated from the first peak (Eccles et al., 1956) of incoming volleys extracted from the CSP (average of all volleys from each nerve; Fig. $1 C$, top) to the onset of the PSTH peak. We adopted a central latency of $<1.5 \mathrm{~ms}$ as a criterion for putative monosynaptic linkage from the SR, DR, or M to the identified SLNs from each nerve (Egger et al., 1986; Moschovakis et al., 1991; Seki et al., 2003). Given the noisy nature of the signal, and the variability in the latency of the CSP across sessions, it is not possible to determine the central latency of spinal neurons in behavioral experiments with the same degree of accuracy as that during acute experiments (e.g., Eccles et al., 1956). Consequently, even though the majority of the analyzed cells are likely to be first-order interneurons with respect to their source nerve, we cannot rule out the possibility that our sample also contains some second-order neurons. In the present paper, neurons with an early response to nerve stimulation are termed SLNs. Putative motoneurons were identified based on their output effect on the EMG signal, and removed from the database. More specifically, if neurons showed a distinct peak in the spike-triggered average of the unrectified EMG signal using only 50 spikes as triggers (Maier et al., 1998) in any of the 10-12 recorded muscles, they were classified as motoneurons and were not analyzed further. Therefore, our dataset reflects all short-latency interneurons from a given primary afferent (e.g., segmental interneurons, ascending projection neurons, or propriospinal neurons) but no motoneurons innervating the wrist or finger muscles recorded in this study.

Characterization of evoked response. For each neuron and stimulated nerve, we generated a PSTH, aligned to the timing of the stimulation pulse (Fig. 1C, bottom). The PSTH included the data from $50 \mathrm{~ms}$ before to $30 \mathrm{~ms}$ after the stimulation pulse, with a bin size of $0.5 \mathrm{~ms}$. The method we used to compute the peak amplitude was similar to that used by Seki et al. (2003), as follows. The baseline firing rate was first computed as the mean bin height in the $50 \mathrm{~ms}$ epoch preceding the stimulation pulse. The peak onset was defined as the time at which the PSTH following the stimulation pulse crossed 2 SDs above the average of the baseline firing rate. Similarly, the peak offset was defined as the time at which the PSTH crossed the above same criterion a second time. To be sure that the algorithm did not detect a local maximum instead of a more visible peak, we used a set of additional ad hoc criteria. First, the peak onset had to occur inside a specific time window (from 0 to $5 \mathrm{~ms}$ after DR or M stimulation, and from 3 to $13 \mathrm{~ms}$ following SR stimulation). Second, the peak had to be $>70 \%$ of the highest bin from the beginning of the PSTH until the end of the detection window. If the detected peak failed to satisfy these ad hoc criteria, the next detected peak was selected, and so on. The peak area was computed as the sum of the bins above baseline firing rate during the peak duration (i.e., see Fig. $1 C$ or Fig. $4 A-C$, gray area). Thus, the peak area can be understood as the mean number of spikes above baseline evoked by each stimulation pulse during the peak time window.

Offline redefinition of task epochs. After identifying the SLNs based on the central latency of orthodromic responses from SR, DR, and M, we evaluated the epoch-dependent modulation of peak area and firing rate during the wrist flexion-extension task. We first redefined each behavioral epoch by analyzing wrist torque and EMG activity onset. Onset and offset of wrist movements (both volitional and passive) were defined based on the smoothed derivative of the wrist torque $(20$ or $50 \mathrm{~Hz}$ lowpass filtered). Movement onset was defined as a rapid and steady change in the derivative of the torque. Movement offset was defined as the first time an arbitrary threshold was crossed following peak movement.

We used the EMG signal to redefine the delay epoch such that the delay preceding the GO signal was devoid of any muscular activity. EMG activity was rectified, aligned with respect to movement onset, averaged across trials, and low-pass filtered at $50 \mathrm{~Hz}$. We computed two thresholds for each EMG signal and used the lower of the two. The first threshold was defined as 5 SDs above the mean EMG amplitude during the rest epoch. The second threshold was defined as $10 \%$ of the difference between the maximum EMG value following movement onset and the mean EMG during rest. We used this dual threshold method to reduce the likelihood of overestimating a threshold value solely based on the rest period, where baseline EMG could be noisy and thus show an artificially large variability (e.g., if nerve stimulation induced muscular twitches).
The EMG onset was defined as the first time point at which the signal stayed above the selected threshold for at least $100 \mathrm{~ms}$. For each given experimental session, we computed the onset of the EMG signal (aligned with respect to movement onset) for each muscle and selected the earliest one from a set of muscles that differed according to the trial type (flexion trials: BRD, FCR, FCU, FDP, FDS, PL, PT, APL; extension trials: ECR, ECU, ED23, ED45, EDC).

After redefining the onset and offset points of movement and EMG activities, we defined five movement-related epochs: (1) rest, $500 \mathrm{~ms}$ interval before onset of the cue signal; (2) delay, from onset of cue signal to the first of either onset of GO signal or earliest EMG onset; (3) active movement, from onset of volitional movement to offset of the movement; (4) active hold, from offset of volitional movement to onset of return signal; and (5) passive movement, from onset of passive return movement to offset of the movement. Only the trials during which the monkey was rewarded, and in which all behavioral events were properly recorded, were analyzed.

Compiling-evoked responses for each behavioral epoch. We measured the evoked response of each IN during the five behavioral epochs listed above. For each IN and each stimulated nerve, the mean evoked response was computed by pooling all the stimulation pulses recorded during the task so as to maximize the signal-to-noise ratio. We then used the onset and offset points of the mean evoked response to compute the peak area evoked in each task epoch (i.e., using the stimulation pulses applied during a specific epoch). We restricted the analysis to those behavioral epochs in which a sufficient number of stimulus pulses were applied, to obtain an unbiased estimate of the evoked response area. The minimum number of stimulation pulses was thus arbitrarily set at 16 stimulations per epoch, as a compromise between the reliability of the peak area and the size of the database. Therefore, the number of analyzed INs differs between epochs. For population analyses, which compared the evoked area of all SLNs across epochs, we normalized and then averaged the peak area in each epoch. For every SLN, normalization was achieved by dividing the peak area in each epoch by the mean evoked response of the neuron (i.e., the area of the response computed by pooling all available stimulation pulses, regardless of task epoch).

Correlation between firing rate and evoked response among behavioral epochs. To assess possible comodulation of evoked response and firing rate with respect to behavior, we performed a correlation analysis between these two variables in single neurons (referred to as "epoch correlation"). For each neuron, we computed the mean firing rate and evoked peak area for each epoch in both movement directions (as in Fig. 4G-I, except that the values at rest were not subtracted), and used Spearman's rank correlation to test the association between firing rate and evoked response. To obtain a fairer estimate of the correlations, we increased the number of data points by including a sixth behavioral epoch, namely, the second rest period following the passive movement (see Fig. 1A). Thus, we examined a maximum of 12 behavioral conditions per neuron, in both this and the following analyses. As indicated in the paragraph above, the number of epochs available for analysis varied between neurons. To guarantee a sufficient number of data points in the present analysis, we only conserved neurons that reached the inclusion criterion (16 or more stimulations per epoch) in at least 5 epochs (of 12). Thus, we assessed the correlation between firing rate and evoked response among the behavioral epochs in $47 \mathrm{DR}$ neurons, $18 \mathrm{M}$ neurons, and 47 SR neurons.

Influence of firing rate on evoked response regardless of behavioral epoch. Although the evoked response could be influenced by external factors (i.e., task epochs), it could also depend on the firing rate of the neuron (as an index of its excitability at the time of stimulation). Therefore, alongside the epoch correlation analysis, we examined the influence of the firing rate of each neuron on its own evoked responses, regardless of behavioral epoch. Stimulation pulses were pooled across task epochs and grouped according to the firing rate of the SLN at the time of stimulation. For each neuron, we sorted individual stimulation pulses into three groups according to the spike count in a $100 \mathrm{~ms}$ window preceding the stimulation (the groups comprised the lowest, median, and highest $33 \%$ of the spike counts). Neurons with $<10$ stimulation pulses per group were removed from the analysis. For each remaining neuron, the evoked response following nerve stimulation was independently computed for 


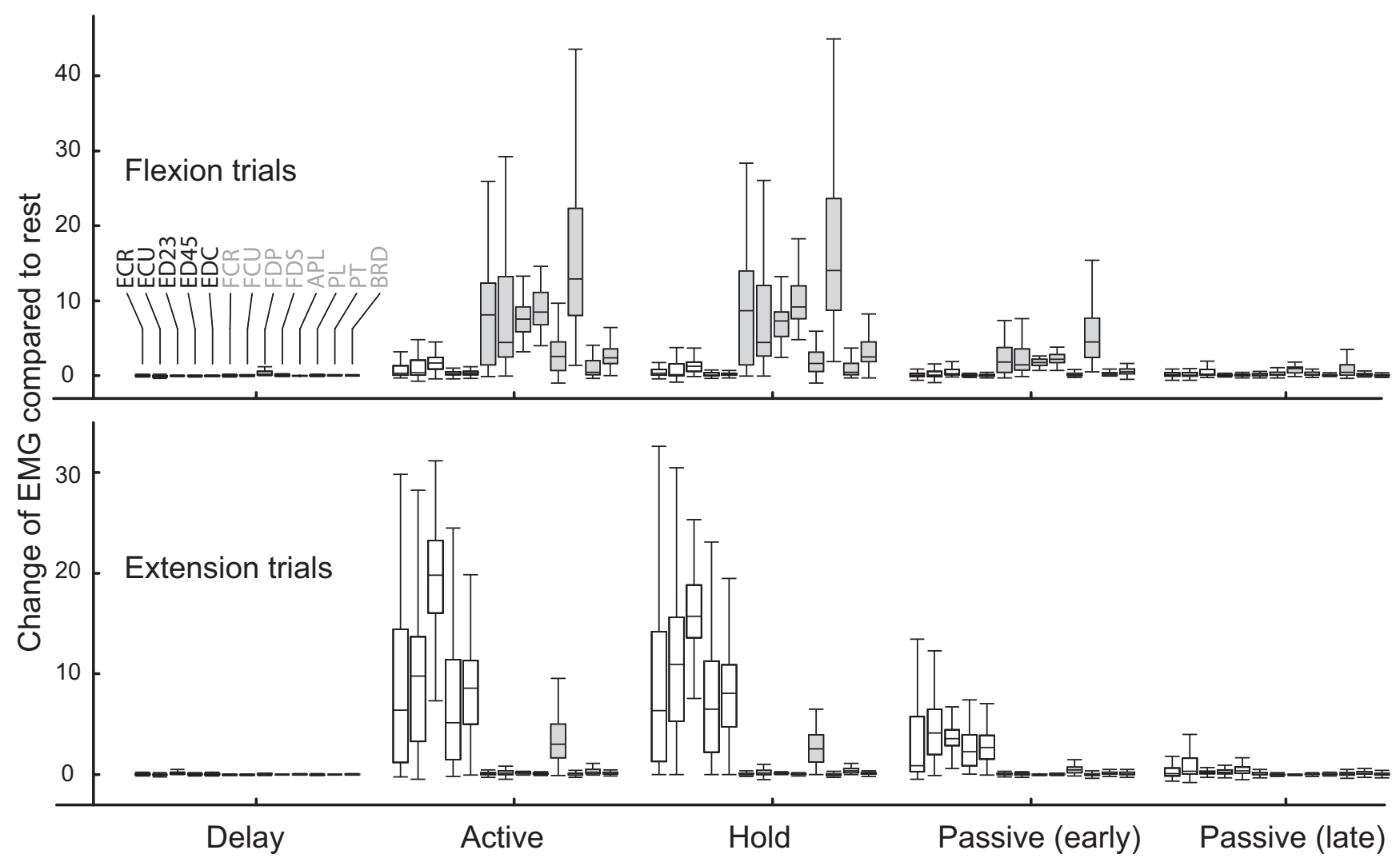

Figure 2. EMG modulation during the behavioral task. RMS of the EMG in different task epochs, expressed as a ratio of the difference in the EMG value compared with that at rest. Box plots represent the median, first, and third quartiles of each EMG. Whiskers indicate nonoutlier extreme data points. Empty boxes represent wrist extensor muscles (names written in black). Filled boxes represent wrist flexors (names written in gray). For the full names of the muscles, see Materials and Methods. The passive movement epoch was divided into early and late halves, and the corresponding EMG values were plotted separately. Top, Flexion trials. Bottom, Extension trials.

each spike count group. We then calculated the difference in evoked response area between the groups with the highest and lowest spike counts.

Because the mean firing rate of a neuron was typically modulated between epochs, we expected the behavioral conditions to indirectly influence our analysis by biasing the distribution of spike counts according to the mean firing rate in the different task epochs. To avoid potential confounds associated with the behavior-driven modulation of firing rate, we restricted the analysis to stimulation pulses applied during behavioral epochs in which we observed a similar mean firing rate. This was achieved by comparing the mean firing rate between epochs (KruskalWallis test) and iteratively eliminating all the stimulation pulses belonging to the epoch with the lowest mean firing rate $(p<0.05)$. When the test failed to reach significance, we assumed that all remaining stimulation pulses were from epochs that had a similar mean firing rate (in the $100 \mathrm{~ms}$ preceding the stimulation).

\section{Results}

\section{EMG modulation during a wrist flexion-extension task}

The aim of the present study was to document the response of spinal neurons to peripheral stimulation during different epochs of a motor task. As a first step, we confirmed that monkeys performed the task as instructed by examining their muscular activity (EMG) in all sessions for which recorded neurons were included in the final database $(n=191$ sessions, EMG signal from 13 muscles in 4 monkeys). The EMG activity was high-pass filtered at $2 \mathrm{~Hz}$ (second order Butterworth filter), and the root mean square (RMS) of the signal was computed and averaged for all task epochs. In all sessions, EMG was expressed as a ratio of the difference between EMG during a specific epoch and EMG during rest (e.g., for the movement epoch: [RMS during move- ment - RMS at rest]/[RMS at rest]). Figure 2 shows the modulation of EMG in 13 muscles compared with rest, pooled across all 4 animals, with the anatomically defined wrist extensors shown in white and wrist flexors shown in gray (Platzer, 2004).

Three aspects of the EMG modulations should be emphasized. First, the EMG signal stayed at rest level during the instructed delay period, indicating that the monkey did not covertly prepare the forthcoming movement by tensing its muscles. Second, the active movement and active hold periods typically involved agonist muscles only (e.g., wrist flexors in flexion trials), with the exception of the APL (a weak wrist flexor) during wrist extension. This indicates that the animals did not cocontract agonist and antagonist muscles in an effort to stiffen their wrist joint against the manipulandum load. Instead, they performed "pure" flexions and extensions during both the active movement and isometric hold phases. Third, during the passive movement epoch, in which the movement direction was opposite to that of the preceding epochs, the EMG activity resembled a downscaled version of that observed during the active movement and hold periods. Thus, the animals may have merely relaxed their agonist muscles at the end of the hold period and passively allowed the springloaded manipulandum to bring their hand back to the rest position, without "helping" it by contracting antagonist muscles. The gradual decrease in EMG activity between the early and late passive movement epochs indicates that the muscle relaxation was not immediate. In conclusion, the monkeys appear to have performed the task as instructed, with relaxed muscles during the instructed Delay period, pure agonist contractions during the 
active phase of the task, and muscle relaxation during the passive movement epoch.

\section{Neuron database}

In total, 388 neurons ( 84 from Monkey KO, 82 from Monkey IS, 87 from Monkey OK, and 135 from Monkey KJ) exhibited a significant response within $10 \mathrm{~ms}$ after the cuff electrode stimulation. Of these, 199 neurons had a central latency (delay between the first peak of the CSP and first bin of the mean evoked response) of $\leq 1.5 \mathrm{~ms}$ and were selected for further analysis. Two of these cells appeared to be motoneurons and were subsequently excluded, such that the final database contained 197 neurons (see Materials and Methods). As explained, we expected most of these neurons to be first-order interneurons: that is, neurons that received monosynaptic connections from one or more of the stimulated nerves. However, as we cannot rule out the possibility that some of these were second-order interneurons, we chose to label them "short-latency neurons" (SLNs) instead of "first-order interneurons" (as in Seki et al., 2003). Of these 197 neurons, we tested 27 for short-latency input from two nerves and 70 for inputs from three nerves. Eight neurons received input from two nerves ( 8 of $97,8 \%$ ), and three neurons received input from three nerves ( 3 of $27,11 \%$ ), leading to a total of 211 nerve-neuron pairs. Among these, eight more neurons were removed from the database because we were unable to clearly define the features of the evoked response (e.g., too few stimulation pulses to generate a reliable estimate of the evoked peak area in any epoch). Thus, the final database contained 203 SLN-nerve pairs (191 neurons): DR, 95 (47\%); M, 56 (27\%); SR, 52 (26\%). Hereafter, we refer to neurons that receive putative monosynaptic input from the DR, $\mathrm{M}$, or SR nerves as "DR neurons," "M neurons," and "SR neurons," respectively.

To estimate the recording depth of these SLNs, we measured the distance between each recording site and the location of the first neuron that we encountered in each penetration (these were expected to be located close to the posterior edge of the dorsal horn). Figure 3 shows a clear difference in recording depth between the SLN groups. When pooling the data from all four monkeys, the median recording depth for SR neurons was $600 \mu \mathrm{m}$ more dorsal than that for the other two SLN groups (KruskalWallis test, $p<0.02$; Tukey-Kramer post hoc tests, $p<0.05$ ). This difference was even more evident when we only considered monkeys in which several nerves were examined (i.e., when we excluded Monkey KJ, in which only SR SLNs were documented). In this case, SR SLNs appear to have been $1 \mathrm{~mm}$ more dorsal than the SLNs from the other two groups (Kruskal-Wallis test, $p<$ 0.006; Tukey-Kramer post hoc tests, $p<0.01$ ). In a previous study, we used the same technique to locate a spinal recording site at a depth of $1490 \mu \mathrm{m}$ below the first cell (Takei and Seki, 2010, their Fig. 7D). Subsequent histological analysis indicated that this site was located around the border of the dorsal horn and intermediate zone. Considering these data, we speculate that the DR SLNs (median depth: $1227 \mu \mathrm{m}$; first-third quartile: 420-1988 $\mu \mathrm{m}$ ) and M SLNs (median depth $1283 \mu \mathrm{m}$; first-third quartile: 604-1842 $\mu \mathrm{m}$ ) in this study were most likely located in the anterior dorsal horn or the intermediate zone (laminae IV-VI). In contrast, the SR SLNs (median depth $645 \mu \mathrm{m}$; first-third quartile: $129-1455 \mu \mathrm{m}$ ) were likely located in a more posterior part of the dorsal horn. That cells receiving cutaneous afferents (i.e., SR SLNs) were located dorsally compared with those receiving muscle afferents (i.e., DR SLNs) is consistent with the known projection patterns of these sensory terminals (Willis and Coggeshall, 2004). Takei and Seki (2010) reported that most premotor in-

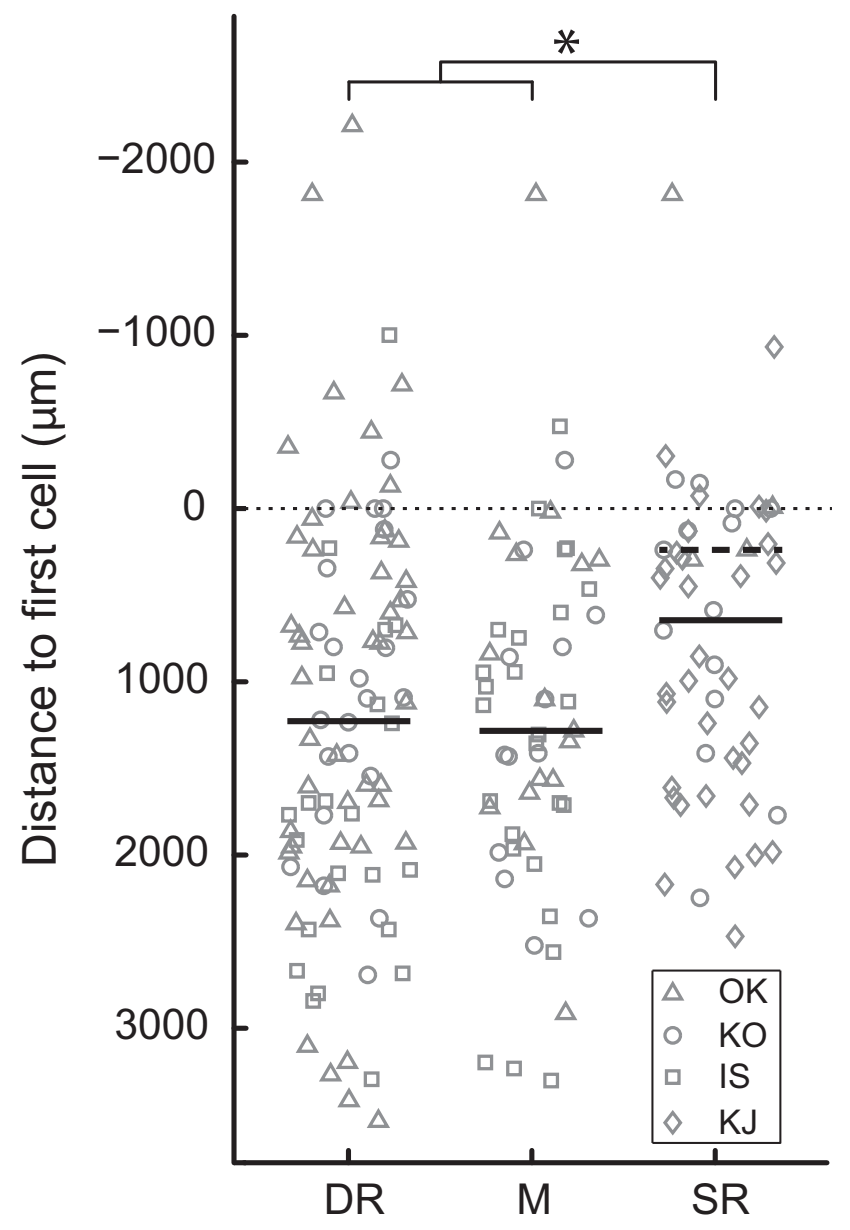

Figure 3. Recording depth relative to the first cell encountered in each penetration. Symbols indicate the animals in which the SLNs were recorded. Diamonds (Monkey KJ) are only present for SR neurons. Solid thick lines indicate the median depth. In SR, the dashed thick line indicates the median depth after excluding the SLNs from Monkey KJ. Negative values (i.e., above the first cell) were typically registered after the electrode reached maximum depth and was being pulled up, most likely displacing spinal tissue (Lemon, 1984). ${ }^{*} p<0.05$ (Kruskal-Wallis test).

terneurons (i.e., monosynaptic to motoneurons) were found between 1500 and $3500 \mu \mathrm{m}$ below the first cell (Takei and Seki, 2010, their Fig. 7A). Thus, there does not seem to be a major overlap between the recording sites of premotor interneurons and our SLNs, which were located more dorsally.

\section{Characteristics of the evoked response}

For each neuron, we generated a PSTH by aligning the neuronal response to all stimulation pulses applied during the task, and assessed the characteristics of the mean evoked response (see Materials and Methods). The rationale of this analysis is based on two assumptions: (1) the peak evoked by stimulation of a nerve reflects the efficacy of the specific connection from the nerve to the recorded neuron; and (2) the baseline firing rate (measured before the stimulation pulse) reflects the gross output of the neuron. In other words, the evoked peak area is an index of a specific input to the neuron, whereas the firing rate is an index of its total output. Examples of such PSTHs are shown for three SLNs in Fig. 4A-C. Among these examples, the DR neuron (Fig. 4A) shows the narrowest peak $(1 \mathrm{~ms})$ and smallest peak area $(0.07 \mathrm{spikes} / \mathrm{stim})$, whereas the SR neuron (Fig. $4 C$ ) shows the longest $(2.5 \mathrm{~ms})$ and largest peak ( 0.48 spikes/stim). As a population, we found that the evoked responses in the spinal SLNs were markedly different 

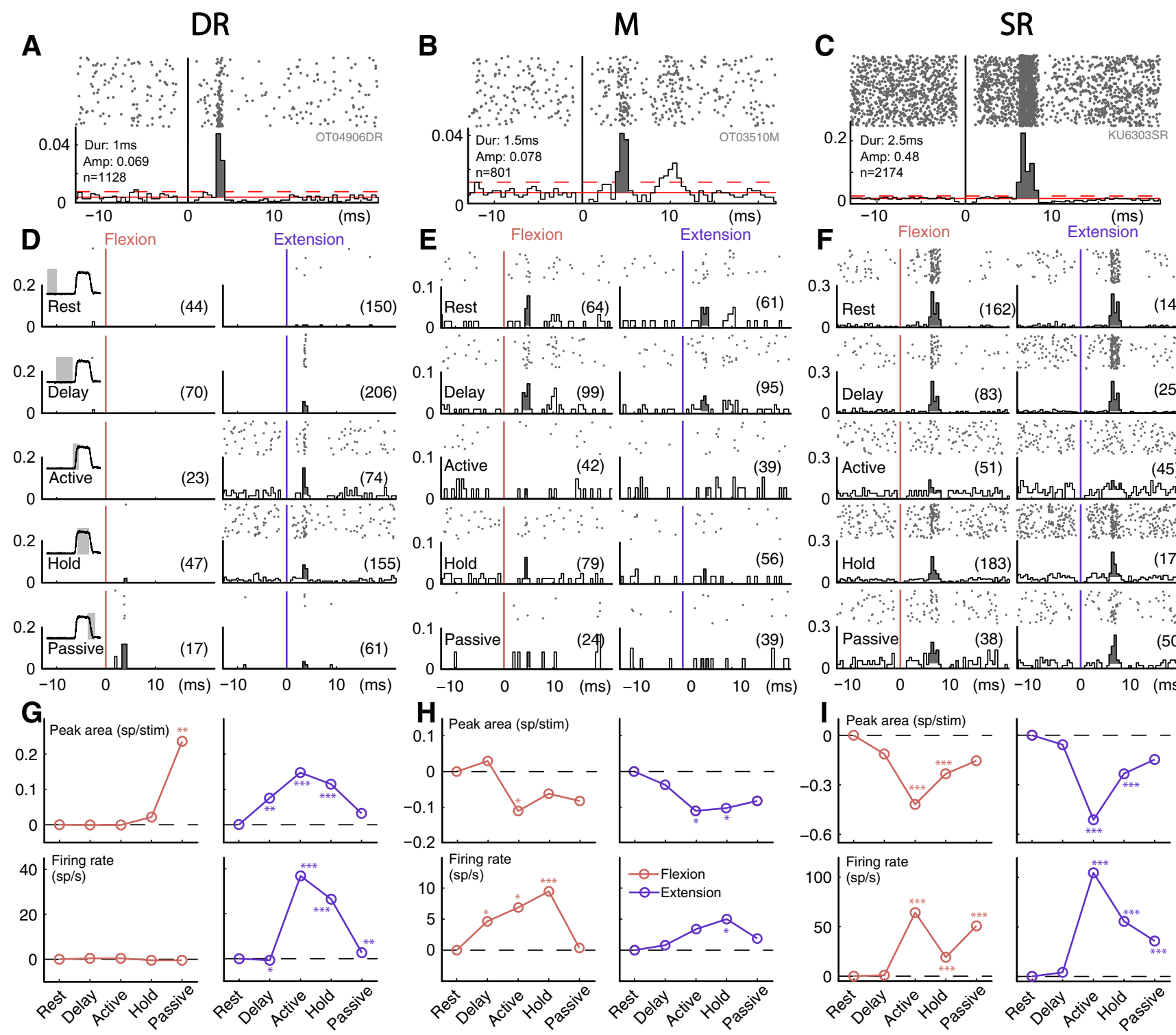

(61)
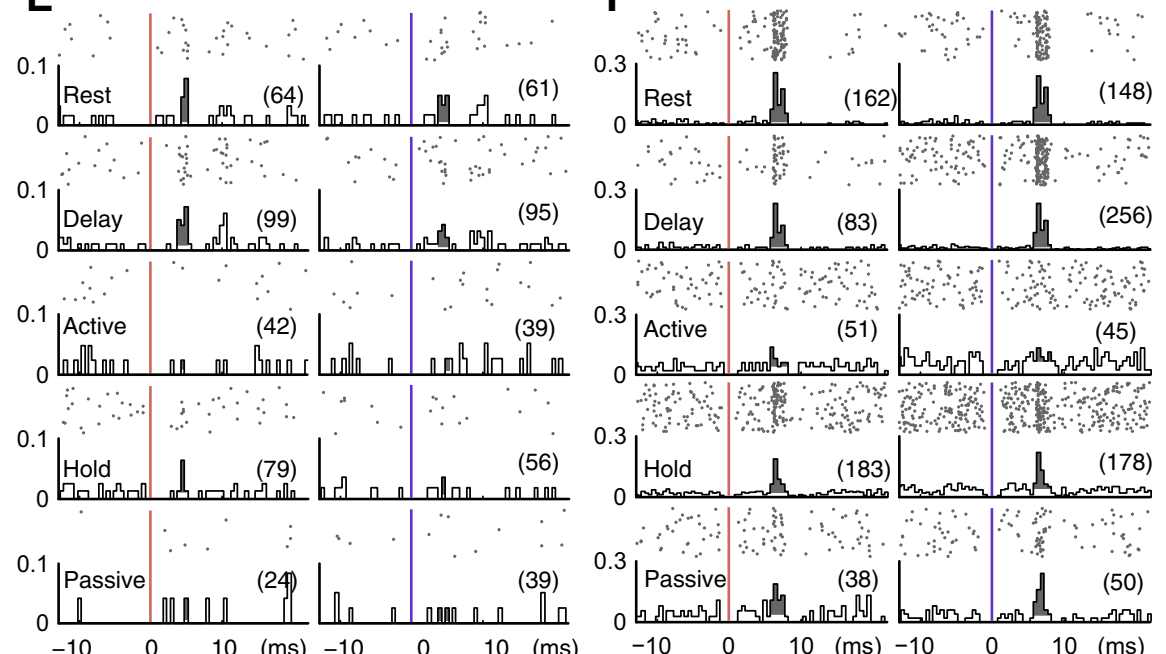

Figure 4. Example of evoked response and firing rate modulation in three SLNs. $\boldsymbol{A}-\boldsymbol{C}$, Raster plot and PSTH of three SLNs using all stimulation pulses applied during the task. PSTH bin size $=0.5 \mathrm{~ms}$. Dur, Duration of the evoked response; Amp, amplitude. All conventions are otherwise similar to Figure $1 \boldsymbol{C}$. $\boldsymbol{D}-\boldsymbol{F}$, Raster plot and PSTH of the three SLNs shown in $\boldsymbol{A}-\boldsymbol{C}$, divided into five behavioral epochs (rows) and two movement directions (columns). Behavioral epochs are illustrated by traces of wrist torque, shown as insets on the left of $\boldsymbol{A}$. Active, Active movement; passive, passive movement. Colored vertical lines indicate the timing of stimulation (red represents flexion trials; blue represents extension trials), and numbers in parentheses indicate the number of stimulation pulses in a given behavioral condition. The peak area represents the gray zone in the PSTH. White part at bottom of the peak represents the baseline mean firing rate preceding the stimulation pulse. $x$-axis indicates time in milliseconds. $y$-axis indicates spiking probability. PSTH bin size $=0.5$ ms. $G-I$, Difference in peak area (top) and mean firing rate (bottom) between rest and the four other behavioral epochs, corresponding to the SLNs shown above. Left plots, Flexion movements (red). Right plots, Extension movements (blue). ${ }^{*} p<0.05$, significant difference in peak area or mean firing rate between rest and another epoch. ${ }^{* *} p<0.01$, significant difference in peak area or mean firing rate between rest and another epoch. ${ }^{* *} p<0.001$ significant difference in peak area or mean firing rate between rest and another epoch. Peak area, Binomial test. Firing rate, Mann-Whitney $\boldsymbol{U}$ test. $\boldsymbol{A}, \boldsymbol{D}, \boldsymbol{G}, \mathrm{DR}$ example neuron. $\boldsymbol{B}, \boldsymbol{E}, \boldsymbol{H}, \mathrm{M}$ example neuron. $\boldsymbol{C}, \boldsymbol{F}, \boldsymbol{I}$, SR example neuron.

Table 1. Mean peak area, duration, and central latency $( \pm S D)$ of evoked responses in SLNs, as well as firing rate at rest ${ }^{a}$

\begin{tabular}{llllc}
\hline & $\begin{array}{l}\text { Peak area } \\
\text { (spikes/stim) }\end{array}$ & $\begin{array}{l}\text { Peak duration } \\
\text { (ms) }\end{array}$ & $\begin{array}{l}\text { Central latency } \\
\text { (ms to (SP) }\end{array}$ & $\begin{array}{l}\text { Firing rate at } \\
\text { rest (Hz) }\end{array}$ \\
\hline DR (95) & $0.14( \pm 0.10)^{a}$ & $1.16( \pm 0.53)^{a}$ & $0.59( \pm 0.38)^{a}$ & $6.75( \pm 8.6)$ \\
M (56) & $0.18( \pm 0.10)^{b}$ & $1.32( \pm 0.61)^{a}$ & $0.82( \pm 0.33)^{b}$ & $6.3( \pm 5.4)$ \\
SR (52) & $0.44( \pm 0.38)^{c}$ & $3.92( \pm 3.24)^{b}$ & $0.99( \pm 0.46)^{b}$ & $4.81( \pm 6.88)$ \\
\hline
\end{tabular}

${ }^{a}$ The numbers in parentheses next to the nerve names indicate the number of analyzed SLNs for each nerve. Superscript letters indicate the different groups, obtained via a Tukey-Kramer post hoc test (one test per column). Groups with different letters showed a significant difference at $p<0.05$, with ascending values in alphabetical order. Groups with the same letter (or no letter) were not statistically different. depending on the stimulated nerve in terms of peak area, duration, and central latency (Table 1). The peak area was smallest for DR, intermediate for M, and largest for SR (Kruskal-Wallis test, $p<10^{-9}$; Tukey-Kramer post hoc tests, $p<0.05$ ). The peak duration was equivalent in DR and $\mathrm{M}$, and shorter in both DR and $\mathrm{M}$ compared with SR (Kruskal-Wallis test, $p<10^{-13}$; Tukey-Kramer post hoc test, $p<10^{-7}$ ). Finally, the central latency of the evoked response was shorter in DR compared with $\mathrm{M}$ and SR neurons (Table 1; Kruskal-Wallis test, $p<10^{-7}$; TukeyKramer post hoc test, $p<0.002)$. In contrast, their mean firing rates at rest were statistically indistinguishable (Kruskal-Wallis test, $p>0.12$ ). 
These results can be partly explained by the types of stimulation used for each nerve. Because DR and M are both sensory and motor nerves, stimulation at high intensity produced muscular twitches that appeared to be aversive to the monkeys. The stimulations used for DR and $\mathrm{M}$ were thus set at only $\sim 1.2$ times the stimulation threshold to avoid causing discomfort to the animal. In contrast, most likely due to the purely cutaneous nature of SR, its stimulation seemed innocuous and elicited no muscle twitch at intensities up to twice the stimulation threshold. Such a large stimulation intensity could generate volleys in many afferent fibers, characterized by various stimulation threshold and conduction velocity (Seki et al., 2009). Furthermore, the large volley could have activated several SLNs that facilitated one another, adding disynaptic or trisynaptic components to the monosynaptic evoked response observed in the recorded neuron. The resulting (compound) evoked responses in SR neurons would thus increase in size and duration.

Physiological differences between nerves may also explain some aspects of the evoked response. The central latency was shorter in DR neurons compared with SR neurons: this result may have been caused by the shorter central latency of the monosynaptic response of spinal neurons to inputs from muscle afferent compared with cutaneous afferents (Aoyama et al., 1988; see also Perreault et al., 1999).

The M neurons also showed a longer central latency compared with DR neurons. A potential explanation is that, because of the clustered distribution of neural fascicles within the median nerve (Stewart, 2003), our stimulation parameters for M neurons (e.g., electrode position, polarity) may have preferentially recruited cutaneous rather than muscle fibers. Another possible explanation for the observed difference in the central latencies of SLNs is that differences in the intrinsic excitability of these neurons led to EPSPs with different sizes or shapes, and thus to PSTHs with different time profiles (Fetz and Gustafsson, 1983).

\section{Modulation of the evoked response during the behavioral task: examples}

We examined whether the evoked response of each SLN was modulated according to behavior, as reported previously (Seki et al., 2003). To specifically assess this modulation, we used the stimulation pulses applied in each behavioral epoch to compute separate PSTHs, and measured the area of the evoked peak for each epoch and each movement direction. We then compared the peak area during the rest epoch with that during the other behavioral epochs for the flexion and extension trials separately. Because, by definition, the duration of the evoked peak of a neuron was identical across behavioral epochs (see Materials and Methods), we converted the peak area in each epoch into a firing probability by dividing it by the peak duration. We then assessed significant differences in peak area between epochs via a binomial test. Figure $4 D-I$ shows representative examples of peak area modulations in a DR neuron (Fig. $4 D, G$ ), an M neuron (Fig. $4 E, H$ ), and an SR neuron (Fig. $4 F, I$ ). We observed striking differences in the modulation of the evoked peak area depending on the source nerve. The DR neuron exhibited a facilitation in firing rate and evoked response compared with rest during the extension trials. More specifically, in the delay epoch, the peak area increased significantly, whereas the firing rate decreased slightly. Subsequently, both firing rate and evoked peak area increased during the active movement and hold epochs. In contrast, the $\mathrm{M}$ neuron showed a completely different pattern. In this example, the firing rate increased during the active part of the task, especially in flexion movements, whereas the evoked response was suppressed in both movement directions. Finally, the SR neuron showed a bidirectional increase in firing rate concomitant with suppression of the evoked peak area during the active movement and hold periods. In summary, Figure 4 shows that example neurons with inputs from cutaneous nerves innervating the dorsal (SR) or palmar (M) aspect of the hand, or proprioceptive afferents innervating wrist flexors (M) or extensors (DR) exhibit different patterns of modulation of their evoked responses during the task. These examples thus illustrate a remarkable dissociation between the modulation of specific input and total output from these neurons.

\section{Modulation of the evoked response during behavioral task: population analysis}

Percentage of modulated neurons compared with rest

We applied the analysis used in Figure $4 G-I$ to all neurons and measured the percentage of neurons that exhibited significant facilitation (upward) or suppression (downward) of peak area (Fig. 5A) and firing rate (Fig. 5B). To ensure that we had fairly estimated the modulation of peak area between the experimental conditions, we only selected neurons that had been stimulated a sufficient number of times in a given behavioral epoch (see Materials and Methods). Consequently, the number of analyzed neurons varies by epoch and depends on the number of stimulation pulses in both the rest period and the considered epoch. Among the 203 FOIN-neuron pairs in our database, 122 (60\%) reached the selection criterion in at least one epoch (54 DR neurons, $27 \mathrm{M}$ neurons, and $47 \mathrm{SR}$ neurons, with 5 neurons receiving more than one input). The mean number of epochs reaching criterion ( \pm SD) for each of these neurons was $9.4 \pm 3.1$ for DR neurons, $8 \pm 4.1$ for M neurons, and $11.2 \pm 1.6$ for SR neurons.

We found that the percentage of neurons with a change of peak area compared with rest was modulated in relation to task epochs, and that the pattern of modulation depended clearly on the source nerve and movement direction (Fig. 5A). Among DR neurons (Fig. 5A, left), few were modulated during the active phase of flexion movement, and neurons with significant evoked peak suppressions and facilitations were found in similar proportion. In contrast, more neurons showed peak facilitation compared with suppression during the active phase of the movement during extension trials ( $\chi^{2}$ test, $p<0.02$ ). For M neurons (Fig. $5 A$, middle), we observed more neurons with peak suppression compared with peak facilitation in both flexion and extension trials. During flexion trials, we found a difference between facilitation and suppression in the active movement epoch $(p<$ 0.02 ). In extension trials, more neurons exhibited suppression compared with facilitation in both the active and passive movement periods (both $p<0.04$ ). Finally, in SR neurons (Fig. 5A, right), the SLN population was biased toward peak suppression during active movement in both flexion $(p<0.006)$ and extension trials $(p<0.02)$, as well as during the hold period of the flexion trials $(p<0.04)$. These results regarding SR neurons contain the same pattern of modulation reported by Seki et al. (2003): during the active part of the task, the evoked response of SR neurons was suppressed. Additionally, these data represent the first evidence that SLNs with input from other nerves do not follow the same pattern of input modulation during movement: whereas the evoked responses of M SLNs tended to be suppressed during flexion and extension movements, the evoked responses of DR SLNs appeared to be modestly facilitated, although this occurred exclusively during extension movements.

In contrast to the peak area, modulation of firing rate (Fig. $5 B$ ) was relatively independent from the source nerve and movement 

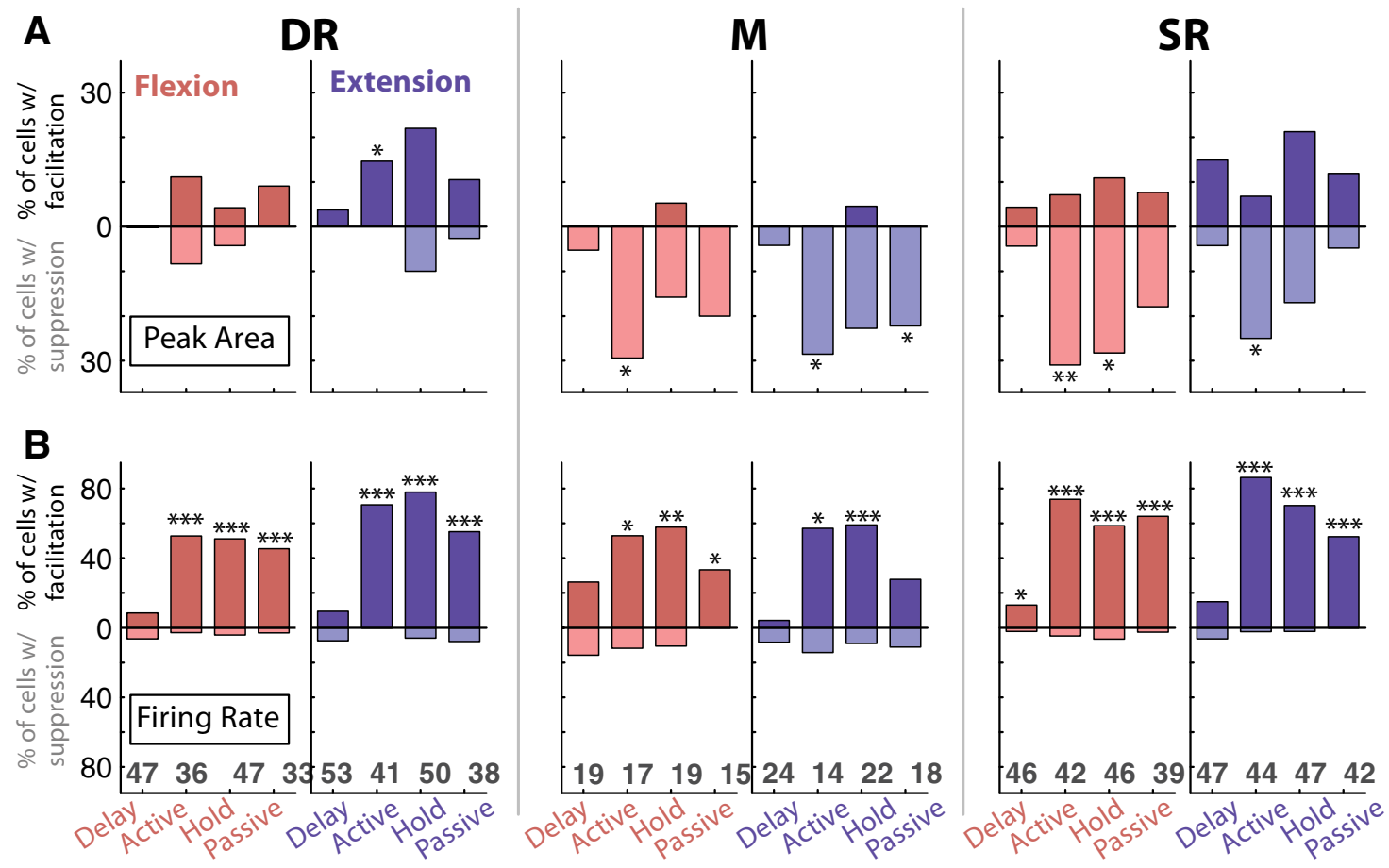

Figure 5. Percentage of SLNs with significantly modulated peak area or firing rate compared with rest. Each plot represents the percentage of SLNs that exhibited a significant modulation of peak area $(\boldsymbol{A})$ or mean firing rate $(\boldsymbol{B})$ compared with rest in the four other task epochs. Top, Darker bars represent the percentage of cells with significant peak area or firing rate facilitation. Bottom, Lighter bars represent peak area or firing rate suppression. Left, Plots (red) represent flexion movements. Right, Plots (blue) represent extension movements. Significant differences were calculated as described in Figure 4G-I. Bottom plots, Numbers at bottom indicate the number of SLNs analyzed in each epoch (this number was identical in $\boldsymbol{A}, \boldsymbol{B})$. ${ }^{*} p<0.05$, proportion of facilitated and suppressed SLNs was significantly different in a specified epoch $\left(\chi^{2}\right.$ test). ${ }^{* *} p<0.01$, proportion of facilitated and suppressed SLNs was significantly different in a specified epoch $\left(\chi^{2}\right.$ test). ${ }^{* * *} p<0.001$, proportion of facilitated and suppressed SLNs was significantly different in a specified epoch ( $\chi^{2}$ test). Leftmost column, DR neurons. Middle column, M neurons. Rightmost column, SR neurons. Active, Active movement; passive, passive movement.

direction, as we observed an increase in firing rate during the task compared with the rest epoch in most of SLNs. The firing rate of spinal SLNs will be fully described in a future publication (S.T. et al., in preparation) and is summarized here for comparison with the evoked response.

Population average of evoked response and firing rate

The input to the SLNs indicated different trends of modulation during the task, depending on the source nerve (Fig. 5). To confirm this result and assess the magnitude of the modulation at the neuronal population level, we analyzed the population average of the peak area and firing rate during the different behavioral epochs. In this analysis, we expressed the peak area of individual cells in each epoch as a ratio of their mean evoked area (i.e., the peak area calculated with all the applied stimulation pulses, see Materials and Methods). For comparison purposes, we applied the same normalization process to firing rate. We refer to these unitless values here as "normalized peak area" (NPA) and "normalized firing rate" (NFR). Unless specified otherwise, we used the Wilcoxon signed-rank test to examine the values in each epoch against those at rest. Figure 6 shows the median (normalized) peak area and firing rate for all the analyzed SLNs in each epoch, separated according to movement direction and source nerve.

The NPA of neurons innervated by different nerves followed distinct patterns of modulation during the task (Fig. 6A), broadly reproducing the results presented in Figure $5 A$. In DR neurons, we only observed a modulatory effect of the NPA in extension trials, whereas it stayed at the rest level during flexion trials (minimum $p$ value in all four epochs: $p>0.36$ ). In contrast, the NPA started to increase during the delay period $(p<0.02)$ in extension trials, and stayed above the rest level during active move- ment $(p<0.03)$ and hold $(p<0.002)$. Even though the number of significantly modulated DR neurons was very low during the delay period in the extension trials ( 2 of 53 facilitations, 0 of 53 suppressions; Fig. $5 A$ ), as a population, the peak area of DR neurons tended to increase compared with that at rest. It is unlikely that a covert movement from the monkey caused this early increase of NPA (e.g., reafference) because, by definition, the EMG level during the delay period stayed at rest level (see Materials and Methods; Fig. 2). This modulation might thus be of descending origin, possibly as a small anticipatory increase in proprioceptive input before and during movement initiation. $M$ neurons showed a consistent suppression of the median NPA during the task in both movement directions. This suppression was significant in flexion trials during the active movement and hold periods ( $p<0.002$ and $p<0.003$, respectively) and in extension trials during the active and passive movement epochs $(p<0.05$ and $p<0.03$, respectively). In SR neurons, the median NPA was suppressed during the active movement and hold periods in the flexion trials ( $p<0.02$ and $p<0.04$, respectively), whereas it was only suppressed during the active movement period in the extension trials $(p<0.03)$.

In contrast, we observed a consistent increase in the NFR during almost all "dynamic" epochs of the task (active movement, hold, passive movement) compared with the rest period in all three SLN populations (Fig. 6B). This result is consistent with our finding that the overwhelming majority of neurons exhibited a significantly facilitated firing rate during the active part of the task compared with rest (Fig. $5 B$ ).

Together, Figures 5 and 6 enable us to make two main conclusions: (1) the efficacy of the input from the three stimulated 

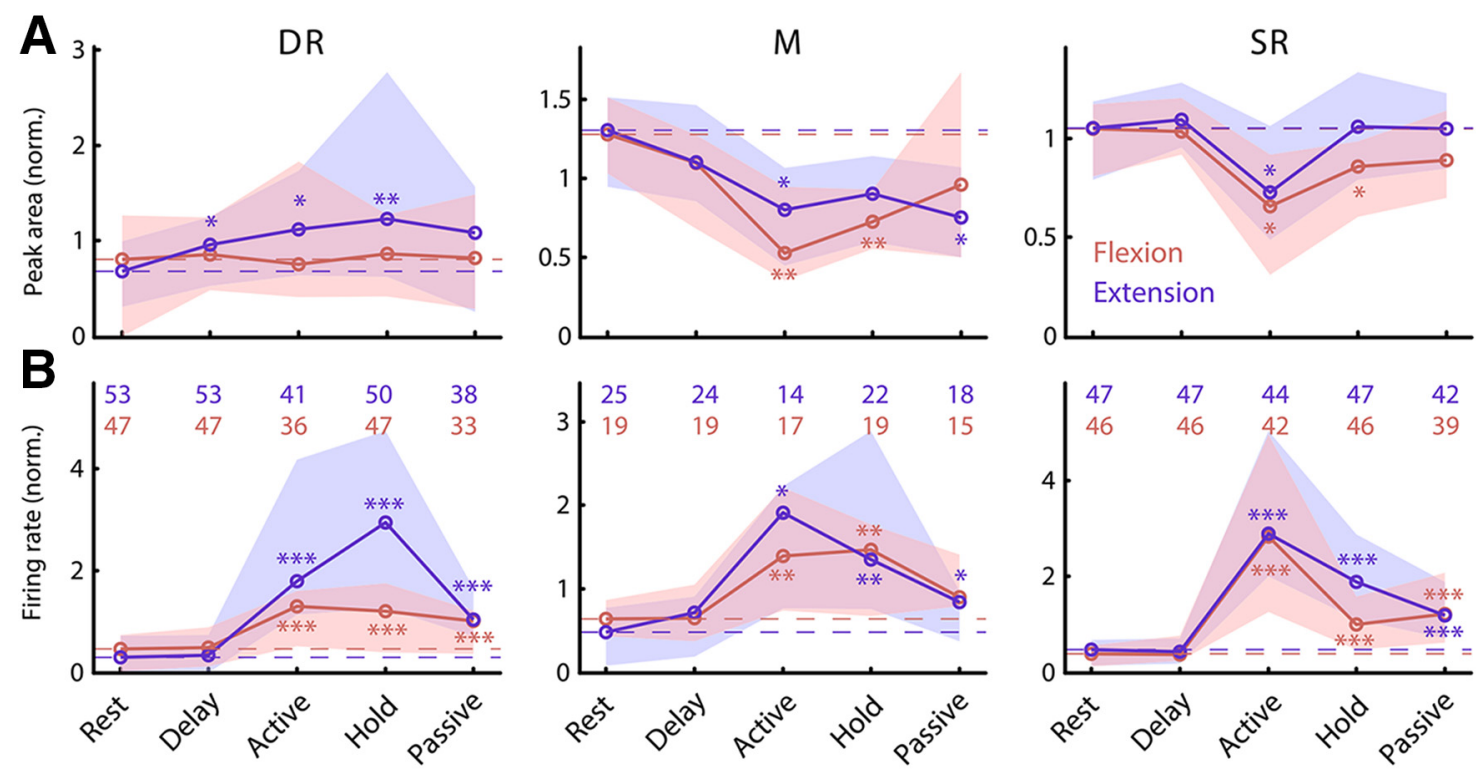

Figure 6. Population measure of evoked response and firing rate modulation. $A$, Median evoked peak area of all analyzed neurons (normalized by their mean peak area, see Results). Red represents flexion trials. Blue represents extension trials. Each column represents a different input nerve, indicated at the top of the figure. Shaded areas represent the interquartile range around each data point. ${ }^{*} p<0.05$, median peak area in a specific epoch was significantly different from that during rest, marked by dashed lines (Wilcoxon signed-rank test). ${ }^{* *} p<0.01$, median peak area in a specific epoch was significantly different from that during rest, marked by dashed lines (Wilcoxon signed-rank test). ${ }^{* *} p<0.001$, median peak area in a specific epoch was significantly different from that during rest, marked by dashed lines (Wilcoxon signed-rank test). $\boldsymbol{B}$, Same as $\boldsymbol{A}$, but the median firing rate is shown. The numbers indicate how many neurons were analyzed for each epoch and direction (red represents flexion; blue represents extension) and are identical in $\boldsymbol{A}, \boldsymbol{B}$. Although the firing rate increased in all epochs compared with rest period in all three neuronal populations, the peak area showed different modulation depending on the source nerve and movement direction.

nerves is modulated at least partially independently from the firing rate of their target neurons (as in Seki et al., 2003); and (2) the input of the three different nerves is distinctly modulated during the behavioral task. Specifically, the input from the DR proprioceptive nerve is facilitated during the delay leading to and during the execution of extension movements; the input from the mixed nerve $\mathrm{M}$ is largely suppressed during both flexion and extension movements; and the input from the cutaneous nerve $\mathrm{SR}$ is suppressed during both flexion and extension movements, and during the active hold period of flexion movements. The regulation of sensory inflow during movement thus seems to depend on the type of peripheral input and on the movement phase or direction, rather than reflecting a general suppression of afferent inputs.

\section{Directionality of the modulation of evoked response and firing rate}

In some neurons, the evoked response was clearly different depending on the movement direction (e.g., Fig. 4G). In contrast, other neurons showed similar modulation during flexion and extension movements (e.g., Fig. 4H,I). To quantify this directionality for the whole neuronal population, we computed the directional difference of the NPA for each neuron (NPA in flexion - NPA in extension). Figure $7 A$ shows the median value of the directional difference of the NPA for the three SLN populations. A value of 0 indicates that the median NPA is equivalent in both movement directions, a positive value indicates a larger NPA for flexion movements, and a negative value indicates a larger value for extension movements. In DR neurons, we observed a larger NPA during the hold period for extension compared with flexion movements $(p<0.02)$. In M neurons, we found no differences in NPA according to movement direction for any epoch. In SR neu- rons, we found a larger NPA during the hold epoch for extension versus flexion trials $(p<0.02)$.

It is noteworthy that the three SLN populations showed similar directionality with respect to modulation of peak area and firing rate. For example, Figure $7 B$ shows that in DR neurons the firing rate was slightly biased toward extension trials during the active movement period $(p<0.03)$, and strongly biased in the same direction during the hold period $(p<0.0006)$. Therefore, in DR neurons, both firing rate and peak area are biased toward wrist extension. In M neurons, no epoch contained any significant directional bias for peak area or firing rate. In SR neurons, the firing rate was larger in extension versus flexion trials during the hold period ( $p<0.003$ ), showing the same bias toward extension as that found for the evoked response. Although this phenomenon could be purely coincidental, alternatively, it may indicate that modulation of firing rate and evoked peak area are not completely independent. We investigate this possibility in the next section.

\section{Relationship between firing rate and peak area modulation}

In theory, the calculation method for the evoked peak area removes any additive influence of firing rate: for each neuron, the baseline firing rate is subtracted from the mean number of spikes evoked by one stimulation pulse (Fig. 1C). Thus, any consistent relationship between firing rate and peak area should not be based simply on the calculation method.

\section{Comodulation of peak area and firing rate with respect to behavioral epochs}

We examined the relationship between firing rate (index of neuronal excitability) and peak area (i.e., neuronal responsiveness) at the single-cell level. We examined whether peak area and baseline firing rate covaried with behavior. We calculated these two vari- 

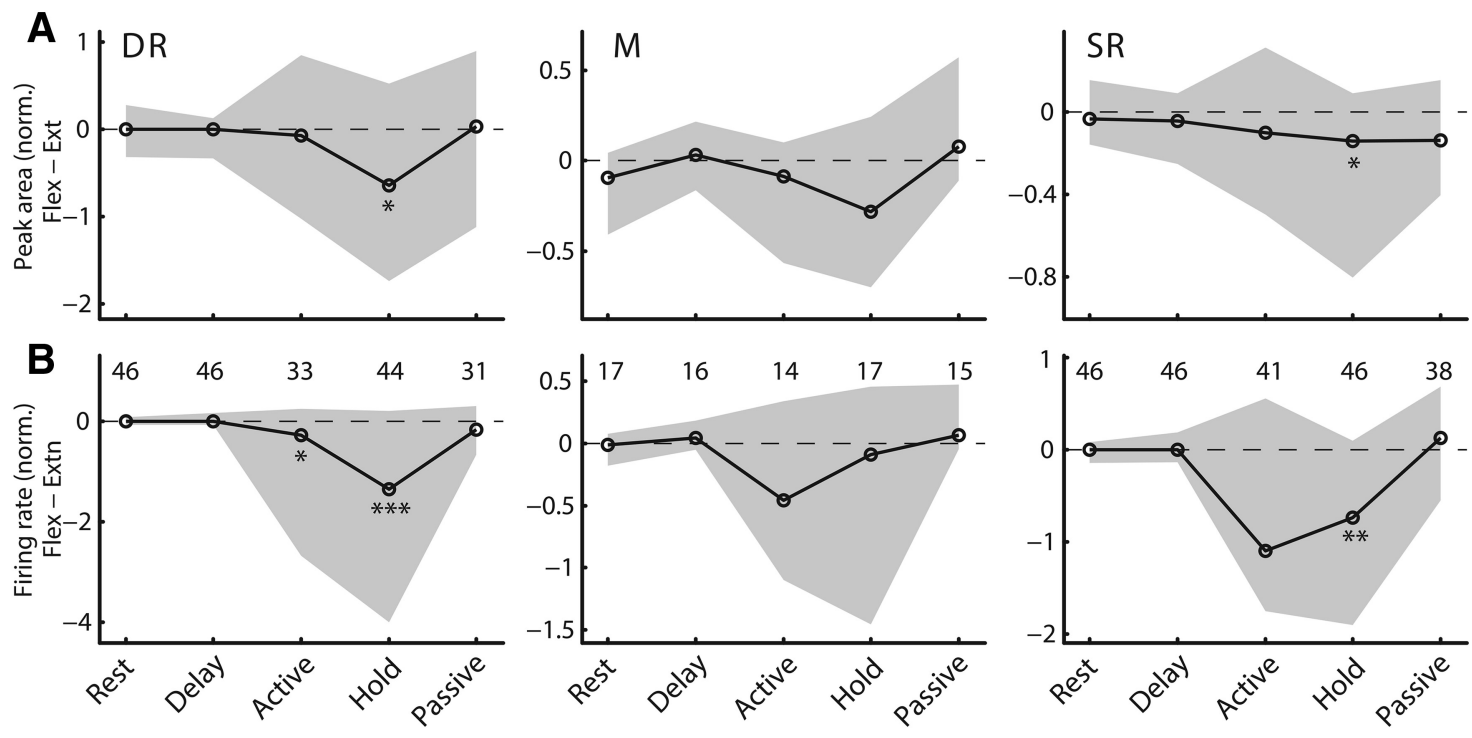

Figure 7. Directionality of evoked response and firing rate. $A$, Median value of the pairwise difference in normalized peak area between flexion and extension trials for all analyzed neurons (a negative value indicates that the peak area was larger in the extension vs flexion trials). Each column represents a different input nerve, indicated at the top of the figure. Shading represents the interquartile around each data point. $\boldsymbol{B}$, Same as $\boldsymbol{A}$, but the difference in normalized firing rate is shown. The numbers indicate how many neurons were analyzed for each epoch and are identical in $\boldsymbol{A}, \boldsymbol{B} .{ }^{*} p<0.05$, significant deviation from 0 , marked by a dashed line (Wilcoxon signed-rank test). ${ }^{* *} p<0.01$, significant deviation from 0 , marked by a dashed line (Wilcoxon signed-rank test). ${ }^{* *} p<0.001$, significant deviation from 0 , marked by a dashed line (Wilcoxon signed-rank test).

ables for each task epoch (as in Fig. 4G-I) and then correlated the variables using Spearman's rank correlation. This analysis was performed on a subset of the database (see Materials and Methods), with a total of 106 neurons ( $86 \%$ of those analyzed in Figs. 5, 6), corresponding to 112 nerve-neuron pairs. The distributions of the correlation coefficients (Spearman's $\rho$ ) are shown in Figure $8 A-C$. We will refer to this analysis as "epoch correlation."

Most of the correlation coefficients were weak, with only a few reaching significance at $p<0.05$ ( 6 of 47, 2 of 18, and 16 of 47 neurons in the DR, M, and SR populations, respectively). The proportion of significant correlations was higher in the SR neurons compared with the two other groups (Barnard's test, DR vs SR: $p<0.009 ; \mathrm{M}$ vs SR: $p<0.05$ ), possibly because of the larger number of epochs available for computation (see Percentage of significantly modulated neurons). Additionally, the median of the coefficient distribution varied greatly between neuronal populations. In DR neurons (Fig. $8 A$ ), the median of the correlation coefficients was strongly biased toward positive values (Wilcoxon sign-rank test vs 0 : $p<$ $0.002)$, whereas it was centered on 0 for the two other nerves $(p>0.68$ and 0.64 in $M$ and SR, respectively).

Focusing on the two epochs in which the modulation of firing rate and peak area was most pronounced across all neurons (active movement and hold; see Figs. $5,6)$, we then examined whether the di-
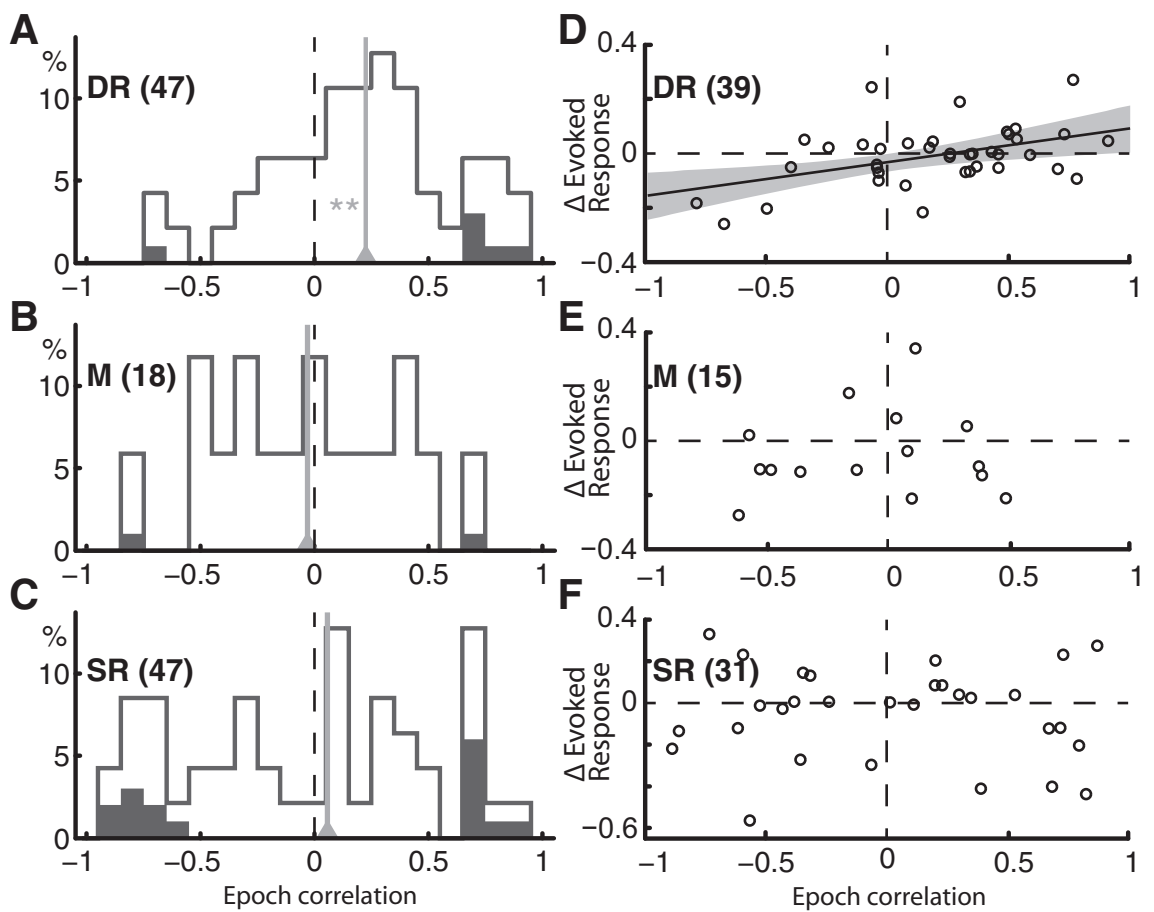

Figure 8. Correlation between evoked peak area and firing rate. $A-C$, Distributions of correlation coefficients (Spearman's rho) between peak area and firing rate across behavioral epochs ("epoch correlation"). Histograms are expressed as percentages of the total number of neurons per population (indicated in parentheses). Filled bins represent significant correlations $(p<0.05)$. The bin size $=0.1$. Dashed line indicates 0 . Light gray line indicates the median value for each population. ${ }^{* *} p<0.01$, significant deviations of the median from 0 (Wilcoxon sign-rank test). Whereas the SR $(\boldsymbol{C})$ and $M$ (B) SLN populations were centered on 0 , the DR population was biased toward a positive value: at the population level, evoked area and firing rates for DR SLNs tended to covary between epochs. $D-F, x$-axis indicates coefficients of the "epoch correlation" shown in $\boldsymbol{A}-\boldsymbol{C}$. $y$-axis indicates difference in peak area between bouts of high and low firing rate (e.g., a negative value represents a "smaller evoked response during periods of high vs low firing rate"). Each dot represents a single neuron. Only DR neurons ( $\boldsymbol{D}$ ) showed a significant positive correlation between these variables (see Results). The straight line and shaded area represent a linear regression and associated $95 \%$ confidence bands. Numbers in parentheses indicate sample size. 


\begin{tabular}{|c|c|c|c|c|c|c|}
\hline & $\begin{array}{l}F R F>E \\
P A F>E\end{array}$ & $\begin{array}{l}F R F>E \\
P A F<E\end{array}$ & $\begin{array}{l}F R F<E \\
P A F>E\end{array}$ & $\begin{array}{l}F R F<E \\
P A F<E\end{array}$ & $\begin{array}{l}\text { Total } \\
N\end{array}$ & $\begin{array}{l}\text { Fisher } \\
p\end{array}$ \\
\hline \multicolumn{7}{|l|}{ DR } \\
\hline Active & 9 & 3 & 5 & 16 & 33 & $0.0091^{* *}$ \\
\hline Hold & 8 & 7 & 6 & 23 & 44 & $0.042^{*}$ \\
\hline \multicolumn{7}{|l|}{ M } \\
\hline Active & 2 & 2 & 2 & 7 & 13 & 0.53 \\
\hline Hold & 5 & 2 & 2 & 7 & 16 & 0.13 \\
\hline \multicolumn{7}{|l|}{ SR } \\
\hline Active & 7 & 12 & 6 & 17 & 42 & 0.52 \\
\hline Hold & 7 & 9 & 9 & 22 & 47 & 0.35 \\
\hline
\end{tabular}

${ }^{a}$ Each row represents the contingency table of one neuronal population (DR, M, SR) in one epoch (Active movement, Hold), with the total number of neurons in each table indicated. $F R$, Firing rate; $P A$, peak area; $F>E$, flexion preferred over extension; $F<E$, extension over flexion.

${ }^{*} p<0.05 ;{ }^{* *} p<0.01$ (significant deviation from independence).

rectional preferences of peak area and firing rate were statistically independent. In both epochs, the neurons were simply classified into two categories according to their directional preference ("larger in flexion" or "larger in extension"), separately for evoked peak area and firing rate. We summarized congruent and incongruent directional preferences for each neuronal population in contingency tables (Table 2). We assessed the statistical independence of the two preference distributions using Fisher's exact test. Table 2 confirms what could be inferred from Figure 7: most of the neurons in the DR and SR populations showed a preference for extension movements, both in terms of peak area and firing rate. However, the contingency tables show a significant deviation from independence in the DR population only, for both analyzed epochs. In other words, the preference of the peak area for flexion or extension trials is predictive of that for firing rate in DR neurons.

In summary, Figure $8 A-C$ and Table 2 indicate that DR SLNs, but neither M nor SR SLNs, tended to show a positive correlation between firing rate and peak area across behavioral conditions (task epochs and movement direction).

Influence of fluctuations in firing rate on the evoked response Membrane potential at the time of stimulation has been shown to affect both the firing rate of a neuron and its responsiveness to external stimulation (Hô and Destexhe, 2000; Haider et al., 2007). The positive correlation between firing rate and peak area shown in DR SLNs could reflect this property, whereas the absence of correlation in M and SR SLN could indicate the influence of external modulation dissociating the output of SLNs from their input (e.g., presynaptic inhibition). Next, we assessed to what extent postsynaptic excitability, as reflected in the firing rate of SLNs, could modulate the evoked response to external stimulations. The association between firing rate and evoked response could either be restricted to large task-dependent modulations of firing rate (i.e., epoch correlation) or could also be influenced by "random" fluctuations in firing rate. The latter case would support the hypothesis that the excitability of the SLNs at the time of stimulation has a visible influence on their evoked response. To examine these possibilities, we checked whether the variation of evoked response based on task-irrelevant fluctuations in firing rate could explain the observed epoch correlation in individual neurons (Fig. $8 D-F$ ).

As the mean firing rate of SLNs typically changes with behavior (Fig. 5B), sorting stimulation pulses according to behavioral epoch or firing rate would make little difference. To limit the influence of behavior-driven changes in mean firing rate, we re- stricted our analysis to include only stimulation pulses applied during epochs with similar mean firing rates (see Materials and Methods). The mean number of behavioral epochs considered for this analysis was $3.0 \pm 2.4$ for DR neurons, $3.2 \pm 2.7$ in M neurons, and $2.8 \pm 3.0$ for SR neurons ( \pm SD, Kruskal-Wallis test, $p>0.27$ ). We pooled the stimulation pulses across the selected behavioral epochs and ordered them by increasing spike number in the $100 \mathrm{~ms}$ window preceding stimulation. We then sorted them into three groups, comprising the stimulations with the $33 \%$ lowest, median, and highest spike counts. The evoked response was computed for each group, and the difference in the peak area between the "high" and "low spike count" groups was calculated. This value represents the difference in neuron responsiveness in situations with high excitability (large spike count) versus low excitability (low spike count). Finally, we compared this value to the "epoch correlation" shown in Figure 8A-C.

Figure $8 D-F$ shows the relationship between the difference in peak area according to background firing rate and the coefficients of the epoch correlation (from Fig. 8A-C). Only DR neurons (Fig. $8 D$ ) showed a positive correlation between these two variables (Spearman's rank correlation, $r=0.35, p<0.03$ ) with a linear slope that was significantly different from $0(y=0.12 x-$ $0.03, p<0.002$ ). In other words, DR SLNs tended to show a similar relationship between evoked response and firing rate, whether it was computed with respect to behavioral epochs or to "random" fluctuations of firing rate.

This linkage between firing rate and evoked response, regardless of task epoch, supports the hypothesis that part of the epoch correlation is mediated by the intrinsic excitability of the SLNs. In contrast, epoch correlation in M and SR neurons does not seem to be related to the excitability of the SLN (Fig. $8 E, F$ ).

Together, these analyses show an interesting pattern of relationships between the firing rate of SLNs and their responsiveness to peripheral nerve stimulation. In neurons receiving projections from the proprioceptive DR nerve, firing rate and evoked response positively comodulated with respect to behavioral epochs. This coupling seemed to be explained in part by the excitability of the SLN at the time of stimulation, as reflected in random fluctuations in firing rate. In contrast, in SLNs receiving projections from the cutaneous SR nerve as well as mixed M nerve, neuronal excitability and responsiveness to peripheral nerve stimulation seemed to be largely independent, suggesting that external sources of modulation may decouple the input and output of the SLNs (e.g., presynaptic inhibition).

\section{Discussion}

Input to spinal neurons from three peripheral nerves of the forearm was modulated during a wrist movement task. The pattern of modulation differed depending on the nerves and, therefore, on the input modality and/or receptive field. Namely, inputs from cutaneous afferents were suppressed while muscle afferents were facilitated. Our data show that peripheral inputs are modulated in a highly dynamic and specific way during motor behavior.

\section{Influence of cutaneous and mixed afferents is suppressed during movement}

We confirmed the results of Seki et al. $(2003,2009)$, who showed that input from the SR to spinal interneurons was presynaptically suppressed during movement. The seemingly independent modulation of SR SLNs and their afferents (Fig. 8) tends to support these results. Tactile sensation is known to decrease during movement (Milne et al., 1988; Chapman, 1994; Duysens et al., 1995; Voss et al., 2006), concurrent with the suppression of cutaneous 
Flexion

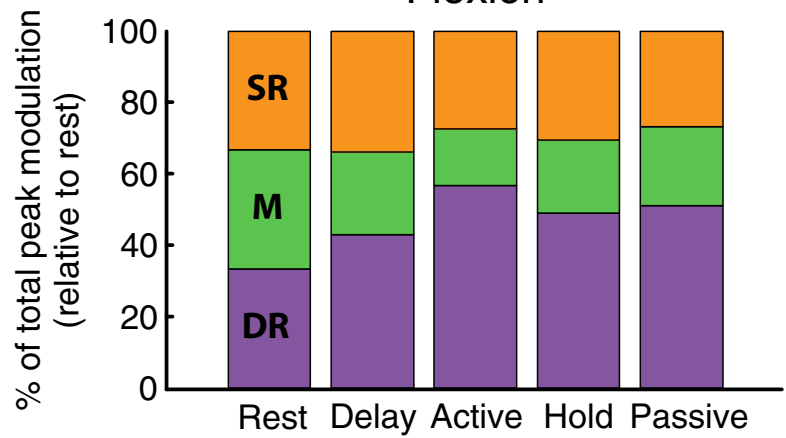

Extension

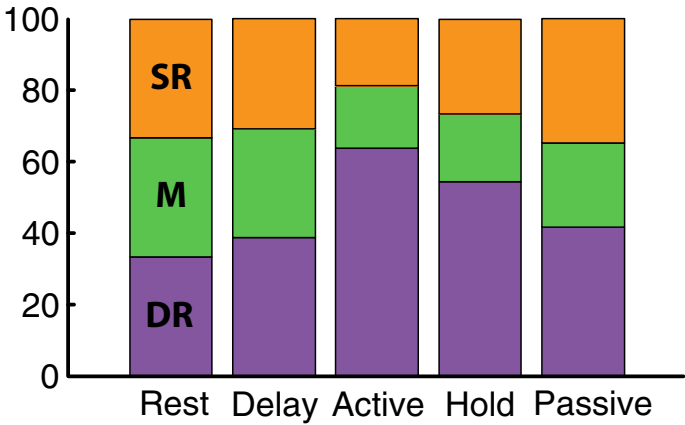

Figure 9. Modulation of the relative input weight from all nerves during behavior. Same data as Figure 6, with a different presentation to illustrate the change of relative importance between peripheral inputs (see Discussion). For each SLN population, the mean evoked peak area in each epoch was divided by the mean evoked peak area at rest. To emphasize the relative importance of the inputs from the three nerves, the mean peak areas of all three nerves are expressed as a percentage of their sum in each epoch. By definition, relative inputs from the three nerves are thus identical during rest (33.3\%). The relative weight of DR input increases with respect to that of the two other nerves as the trial progresses. This phenomenon is especially visible in extension trials, which involve a contraction of DR spindle-bearing muscles.

peripheral signals (Ghez and Pisa, 1972; Chapin et al., 1982; Jiang et al., 1990, 1991; Seki and Fetz, 2012). Furthermore, cutaneous input is known to strongly modulate motor output, for example, through a powerful cutaneous motor reflex (Bui et al., 2013). Thus, unregulated cutaneous inputs generated by movement might disturb motor commands by generating improper reafferent signals. The (likely presynaptic) suppression of input from cutaneous and mixed nerves might reflect one of the mechanisms by which the CNS regulates input to spinal and supraspinal centers.

The modulation of responsiveness in M SLNs was somewhat unexpected. Because the median nerve is a mixed nerve with both cutaneous and muscle afferents, we expected results halfway between that of DR (proprioceptive) and SR (cutaneous) SLNs. However, as shown in Figures 6 and 8, the M results closely resembled those of SR SLNs. One possibility is that our sample of M SLNs was biased toward cutaneous fibers and that the same mechanisms influenced both SR and M inputs. Three elements support this idea. First, the firing rate of M SLNs was symmetrical during flexion and extension, which more closely resembles the pattern of SR compared with that of DR (Fig. 6). Second, the depression of the monosynaptic response was equivalent during both flexion and extension trials. Such nondirectional suppression was described as a typical feature of cutaneous gating in several reports (Chapman et al., 1988; Jiang et al., 1990, 1991; Seki and Fetz, 2012). Third, the central latency of the evoked response in $\mathrm{M}$ neurons was longer than that in DR and equivalent to that in SR neurons (Table 1 ). This could mean that $M$ neurons received a dominant input from slowly conducting cutaneous fibers (Aoyama et al., 1988).

\section{Influence of afferents from wrist extensor muscles is facilitated during active wrist extension}

Classical works have shown that presynaptic inhibition of sensory afferents can be selective with respect to different fiber types ( $\mathrm{Ru}$ domín et al., 1983) or even different branches of the same afferent (Lomelí et al., 1998). Our findings represent the first evidence of how this potential for specific modulation can be used during motor behavior. Indeed, unlike SR and M SLNs, DR neurons showed facilitation of both evoked responses and firing rate during wrist extension movements, that is, only when their spindlebearing muscles underwent a shortening contraction. This result indicates that, during motor behavior, the input and output of muscle afferent SLNs do not passively reflect the lengthening of their spindle-bearing muscles.

Unlike M and SR neurons, we found that, for DR neurons, the modulation of firing rate and evoked response was somehow correlated, even after removing the influence of behavioral epochs (Fig. 8). This coupling could be achieved by enhancing the transmission efficacy between primary afferents and postsynaptic cells (Powers and Binder, 1995) or by modulating the recruitment gain of postsynaptic cells by primary afferents (Kernell and Hultborn, 1990). For example, as with a decrease in input resistance during fictive locomotion (Shefchyk and Jordan, 1985), the membrane potential of postsynaptic cells might be adjusted to a level within their dynamic range for action potential generation (Schwindt and Crill, 1982). In this way, a fixed synaptic input from primary afferents could recruit postsynaptic neurons more effectively. Interestingly, responsiveness to afferent inputs in DR SLNs increased before EMG onset (Fig. 6), suggesting that gain control might precede the start of movement. If so, DR SLNs could quickly transmit or even amplify the influence of proprioceptive inputs generated by the contraction of their spindlebearing muscles. From the standpoint of descending motor commands, taking advantage of highly active primary afferents during movement to drive postsynaptic neurons would be an efficient strategy.

Input from muscle afferents to the spinal cord is regulated by presynaptic inhibition from both descending and segmental inputs (Rudomín et al., 1983, 1986). Presynaptic modulation of muscle afferents projecting to limb motoneurons has also been extensively studied in humans by means of refined reflex testing (Pierrot-Deseilligny and Burke, 2012). These studies indicate that descending cortical influence increases the postsynaptic inhibition of Ia afferents in the forelimb (Meunier and PierrotDeseilligny, 1998), whereas peripheral cutaneous input suppress this inhibition (Berardelli et al., 1987; Nakashima et al., 1990; Iles, 1996; Aimonetti et al., 1999, 2000). This presynaptic control may play a role in stabilizing a limb during movement (Capaday and Stein, 1987a, 1989) or facilitate the transition from posture to movement (Crevecoeur and Scott, 2014). Although it is somewhat counterintuitive given the literature on the topic, we found that spinal interneurons with direct input from muscle afferents showed increased evoked responses during movement (Fig. 6). Although muscle afferents undergo strong presynaptic inhibition that prevents them from threatening the stability of motor output 
(Fink et al., 2014), this result could be construed as evidence that a subtle reduction of presynaptic inhibition may occur during voluntary movement. This relative decrease in presynaptic inhibition could explain why we observed some signs of postsynaptic modulation of the evoked response (Fig. 8D). The resulting increase in spinal responsiveness during extension trials might act as servo-assistance (Windhorst, 2007) and slightly enhance the reflex gain during an epoch requiring sustained motor output. Increased SLN responsiveness might also improve the transmission of proprioceptive information to supraspinal centers, which are particularly critical during the active part of the task (Scott, 2012). A recent study reported that modulation of the fusimotor drive could facilitate proprioceptive input during motor learning (Dimitriou, 2016). Here, we show another way by which the CNS might regulate the balance between peripheral inputs during motor behavior.

\section{Coupling the input from primary afferents with the output of spinal first-order interneurons}

The modulation of peripheral inputs to a spinal SLN could be coupled or uncoupled with respect to firing rate. The DR SLNs in this study exemplify such coupling: during active extension, their firing rate and response to nerve stimulations were both facilitated. Because all monosynaptic inputs from primary afferents to SLNs are excitatory, any DR input under this coupling mode is expected to facilitate the SLNs to increase their total output, thus enhancing the relative weight of inputs from DR on the overall activity of spinal networks. Such coupling can be accomplished by decreasing the influence of presynaptic inhibition or by increasing the input gain of the SLNs. In contrast, decoupling was illustrated by the SR SLNs, in which the response evoked by peripheral input was significantly suppressed while the total output was consistently facilitated. Under this mode, sensory input from the SR is not expected to optimally assist the SLNs to increase their total output. Such uncoupling could be the typical result of a strong influence of presynaptic inhibition. Importantly, this state of coupling or uncoupling between primary afferent and postsynaptic SLNs not only is specific to the input nerve but also changes dynamically according to behavior. For example, uncoupling between SR afferents and their SLNs is evident during active, but not passive, movement (Fig. 6). Thus, the transmission between primary afferents and SLNs may exhibit dynamic transitions between functional coupling and decoupling, depending on behavior. This mechanism might act as an early "switching board," dynamically facilitating and suppressing different sensory sources to alter the relative balance of their influence on spinal networks and, subsequently, the CNS. This idea is illustrated by Figure 9, which shows the same data as Figure $6 \mathrm{~A}$ in another form. As the trial progresses, the weight of proprioceptive inputs increases compared with that of other nerves, especially in extension trials. The total somatosensory input to a specific segment of the spinal cord could thus be thought of as weighted a sum of various inputs, the weights of which would be determined by their relevance to the task being performed.

In conclusion, the influence of peripheral afferents on spinal interneurons is specifically modulated, depending on modality (cutaneous vs muscle afferents) and the motor behavior of the animal (contraction vs relaxation of spindle-bearing muscles). Our data indicate that spinal first-order interneurons might act as transmission relays, the activity of which could be coupled or uncoupled from peripheral inputs during voluntary movement. Such coupling modulation is likely to be mainly mediated by presynaptic inhibition but may also be influenced by gain control of postsynaptic neurons.

\section{References}

Aimonetti JM, Schmied A, Vedel JP, Pagni S (1999) Ia presynaptic inhibition in human wrist extensor muscles: effects of motor task and cutaneous afferent activity. J Physiol Paris 93:395-401. CrossRef Medline

Aimonetti JM, Vedel JP, Schmied A, Pagni S (2000) Mechanical cutaneous stimulation alters Ia presynaptic inhibition in human wrist extensor muscles: a single motor unit study. J Physiol 522:137-145. CrossRef Medline

Angel RW, Malenka RC (1982) Velocity-dependent suppression of cutaneous sensitivity during movement. Exp Neurol 77:266-274. CrossRef Medline

Aoyama M, Hongo T, Kudo N (1988) Sensory input to cells of origin of uncrossed spinocerebellar tract located below Clarke's column in the cat. J Physiol 398:233-257. CrossRef Medline

Baken BC, Nieuwenhuijzen PH, Bastiaanse CM, Dietz V, Duysens J (2006) Cutaneous reflexes evoked during human walking are reduced when selfinduced. J Physiol 570:113-124. CrossRef Medline

Bays PM, Flanagan JR, Wolpert DM (2006) Attenuation of self-generated tactile sensations is predictive, not postdictive. PLoS Biol 4:e28. CrossRef Medline

Berardelli A, Day BL, Marsden CD, Rothwell JC (1987) Evidence favouring presynaptic inhibition between antagonist muscle afferents in the human forearm. J Physiol 391:71-83. CrossRef Medline

Bui TV, Akay T, Loubani O, Hnasko TS, Jessell TM, Brownstone RM (2013) Circuits for grasping: spinal dI3 interneurons mediate cutaneous control of motor behavior. Neuron 78:191-204. CrossRef Medline

Capaday C, Stein RB (1987a) A method for simulating the reflex output of a motoneuron pool. J Neurosci Methods 21:91-104. CrossRef Medline

Capaday C, Stein RB (1987b) Difference in the amplitude of the human soleus H reflex during walking and running. J Physiol 392:513-522. CrossRef Medline

Capaday C, Stein RB (1989) The effects of postsynaptic inhibition on the monosynaptic reflex of the cat at different levels of motoneuron pool activity. Exp Brain Res 77:577-584. Medline

Chapin JK, Woodward DJ (1982) Somatic sensory transmission to the cortex during movement: gating of single cell responses to touch. Exp Neurol 78:654-669. CrossRef Medline

Chapman CE (1994) Active versus passive touch: factors influencing the transmission of somatosensory signals to primary somatosensory cortex. Can J Physiol Pharmacol 72:558-570. CrossRef Medline

Chapman CE, Jiang W, Lamarre Y (1988) Modulation of lemniscal input during conditioned arm movements in the monkey. Exp Brain Res 72: 316-334. Medline

Collins DF, Cameron T, Gillard DM, Prochazka A (1998) Muscular sense is attenuated when humans move. J Physiol 508:635-643. CrossRef Medline

Confais J, Kim G, Tomatsu T, Takei T, Seki K (2015) Differential modulation of sensory input from three forearm afferent nerves to the spinal cord of the primate during delayed wrist movements. Soc Neurosci Abstr $516.08 / \mathrm{R} 12$

Crevecoeur F, Scott SH (2014) Beyond muscles stiffness: importance of state-estimation to account for very fast motor corrections. PLoS Comput Biol 10:e1003869. CrossRef Medline

Dimitriou M (2016) Enhanced muscle afferent signals during motor learning in humans. Curr Biol 26:1-7. CrossRef Medline

Duysens J, Tax AA, Nawijn S, Berger W, Prokop T, Altenmüller E (1995) Gating of sensation and evoked potentials following foot stimulation during human gait. Exp Brain Res 105:423-431. Medline

Eccles JC, Fatt P, Landgren S (1956) Central pathway for direct inhibitory action of impulses in largest afferent nerve fibres to muscle. J Neurophysiol 19:75-98. Medline

Egger MD, Freeman NC, Jacquin M, Proshansky E, Semba K (1986) Dorsal horn cells in the cat responding to stimulation of the plantar cushion. Brain Res 383:68-82. CrossRef Medline

Fetz EE, Gustafsson B (1983) Relation between shapes of post-synaptic potentials and changes in firing probability of cat motoneurones. J Physiol 341:387-410. CrossRef Medline

Fink AJ, Croce KR, Huang ZJ, Abbott LF, Jessell TM, Azim E (2014) Presynaptic inhibition of spinal sensory feedback ensures smooth movement. Nature 509:43-48. CrossRef Medline 
Ghez C, Pisa M (1972) Inhibition of afferent transmission in cuneate nucleus during voluntary movement in the cat. Brain Res 40:145-155. CrossRef Medline

Haider B, Duque A, Hasenstaub AR, Yu Y, McCormick DA (2007) Enhancement of visual responsiveness by spontaneous local network activity in vivo. J Neurophysiol 97:4186-4202. CrossRef Medline

Haugland M (1996) A flexible method for fabrication of nerve cuff electrodes, Vol 1, pp 359-360. 18th Annual International Conference of the IEEE Engineering in Medicine and Biology Society, Amsterdam.

Hô N, Destexhe A (2000) Synaptic background activity enhances the responsiveness of neocortical pyramidal neurons. J Neurophysiol 84:14881496. Medline

Iles JF (1996) Evidence for cutaneous and corticospinal modulation of presynaptic inhibition of Ia afferents from the human lower limb. J Physiol 491:197-207. CrossRef Medline

Jiang W, Lamarre Y, Chapman CE (1990) Modulation of cutaneous cortical evoked potentials during isometric and isotonic contractions in the monkey. Brain Res 536:69-78. CrossRef Medline

Jiang W, Chapman CE, Lamarre Y (1991) Modulation of the cutaneous responsiveness of neurones in the primary somatosensory cortex during conditioned arm movements in the monkey. Exp Brain Res 84:342-354. Medline

Kernell D, Hultborn H (1990) Synaptic effects on recruitment gain: a mechanism of importance for the input-output relations of motoneurone pools? Brain Res 507:176-179. CrossRef Medline

Lemon R (1984) IBRO handbook series: methods in the neuroscience, Vol 4. Methods for neuronal recording in conscious animals. Chichester, United Kingdom: Wiley.

Lomelí J, Quevedo J, Linares P, Rudomín P (1998) Local control of information flow in segmental and ascending collaterals of single afferents. Nature 395:600-604. CrossRef Medline

Maier MA, Perlmutter SI, Fetz EE (1998) Response patterns and force relations of monkey spinal interneurons during active wrist movement. J Neurophysiol 80:2495-2513. Medline

Meunier S, Pierrot-Deseilligny E (1998) Cortical control of presynaptic inhibition of Ia afferents in humans. Exp Brain Res 119:415-426. CrossRef Medline

Milne RJ, Aniss AM, Kay NE, Gandevia SC (1988) Reduction in perceived intensity of cutaneous stimuli during movement: a quantitative study. Exp Brain Res 70:569-576. Medline

Moschovakis AK, Sholomenko GN, Burke RE (1991) Differential control of short latency cutaneous excitation in cat FDL motoneurons during fictive locomotion. Exp Brain Res 83:489-501. Medline

Nakashima K, Rothwell JC, Day BL, Thompson PD, Marsden CD (1990) Cutaneous effects on presynaptic inhibition of flexor Ia afferents in the human forearm. J Physiol 426:369-380. CrossRef Medline

Palmer CE, Davare M, Kilner JM (2016) Physiological and perceptual sensory attenuation have different underlying neurophysiological correlates. J Neurosci 36:10803-10812. CrossRef Medline

Perreault MC, Shefchyk SJ, Jimenez I, McCrea DA (1999) Depression of muscle and cutaneous afferent-evoked monosynaptic field potentials during fictive locomotion in the cat. J Physiol 521:691-703. CrossRef Medline
Pierrot-Deseilligny E, Burke D (2012) Presynaptic inhibition of Ia terminals. In: The circuitry of the human spinal cord, pp 292-333. Cambridge, United Kingdom: Cambridge UP.

Platzer W (2004) Color atlas of human anatomy, Vol 1. Locomotor system. Stuttgart: Thieme.

Powers RK, Binder MD (1995) Effective synaptic current and motoneuron firing rate modulation. J Neurophysiol 74:793-801. Medline

Prut Y, Fetz EE (1999) Primate spinal interneurons show pre-movement instructed delay activity. Nature 401:590-594. CrossRef Medline

Rudomín P (2002) Selectivity of the central control of sensory information in the mammalian spinal cord. Adv Exp Med Biol 508:157-170. CrossRef Medline

Rudomín P, Schmidt RF (1999) Presynaptic inhibition in the vertebrate spinal cord revisited. Exp Brain Res 129:1-37. CrossRef Medline

Rudomín P, Jiménez I, Solodkin M, Dueñas S (1983) Sites of action of segmental and descending control of transmission on pathways mediating PAD of Ia- and Ib-afferent fibers in cat spinal cord. J Neurophysiol 50: 743-769. Medline

Rudomín P, Solodkin M, Jiménez I (1986) PAD and PAH response patterns of group Ia- and Ib-fibers to cutaneous and descending inputs in the cat spinal cord. J Neurophysiol 56:987-1006. Medline

Rushton DN, Rothwell JC, Craggs MD (1981) Gating of somatosensory evoked potentials during different kinds of movement in man. Brain 104:465-491. CrossRef Medline

Schwindt PC, Crill WE (1982) Factors influencing motoneuron rhythmic firing: results from a voltage-clamp study. J Neurophysiol 48:875-890.

Scott SH (2012) The computational and neural basis of voluntary motor control and planning. Trends Cogn Sci 16:541-549. CrossRef Medline

Seki K, Fetz EE (2012) Gating of sensory input at spinal and cortical levels during preparation and execution of voluntary movement. J Neurosci 32:890-902. CrossRef Medline

Seki K, Perlmutter SI, Fetz EE (2003) Sensory input to primate spinal cord is presynaptically inhibited during voluntary movement. Nat Neurosci 6:1309-1316. CrossRef Medline

Seki K, Perlmutter SI, Fetz EE (2009) Task-dependent modulation of primary afferent depolarization in cervical spinal cord of monkeys performing an instructed delay task. J Neurophysiol 102:85-99. CrossRef Medline

Shefchyk SJ, Jordan LM (1985) Motoneuron input-resistance changes during fictive locomotion produced by stimulation of the mesencephalic locomotor region. J Neurophysiol 54:1101-1108. Medline

Stewart JD (2003) Peripheral nerve fascicles: anatomy and clinical relevance. Muscle Nerve 28:525-541. CrossRef Medline

Takei T, Seki K (2010) Spinal interneurons facilitate coactivation of hand muscles during a precision grip task in monkeys. J Neurosci 30:1704117050. CrossRef Medline

Voss M, Ingram JN, Haggard P, Wolpert DM (2006) Sensorimotor attenuation by central motor command signals in the absence of movement. Nat Neurosci 9:26-27. CrossRef Medline

Willis WD, Coggeshall RE (2004) Sensory mechanisms of the spinal cord, Ed 3, Vol 1. Primary afferent neurons and the spinal dorsal horn. New York: Kluwer Academic/Plenum.

Windhorst U (2007) Muscle proprioceptive feedback and spinal networks. Brain Res Bull 73:155-202. CrossRef Medline 